MATHEMATICS OF COMPUTATION

Volume 75, Number 256, October 2006, Pages 1809-1831

S 0025-5718(06)01860-6

Article electronically published on June 6, 2006

\title{
NUMERICAL APPROXIMATIONS OF THE 10-MOMENT GAUSSIAN CLOSURE
}

\author{
CHRISTOPHE BERTHON
}

\begin{abstract}
We propose a numerical scheme to approximate the weak solutions of the 10-moment Gaussian closure. The moment Gaussian closure for gas dynamics is governed by a conservative hyperbolic system supplemented by entropy inequalities whose solutions satisfy positiveness of density and tensorial pressure. We consider a Suliciu-type relaxation numerical scheme to approximate the solutions. These methods are proved to satisfy all the expected positiveness properties and all the discrete entropy inequalities. The scheme is illustrated by several numerical experiments.
\end{abstract}

\section{INTRODUCTION}

Currently, many of the numerical simulations for compressible flows are proposed within the framework of Euler equations. This system, related to velocity moments of the Boltzmann equation, is based on the assumption that the gas is in local thermodynamic equilibrium. Several recent applications consider a gas which moves away from equilibrium. Since the main assumption is not satisfied, the classical Euler equations cannot be used.

The computations of extremely low pressure rarefied gas flows from the reentry of space vehicles enter the present framework where the local thermodynamic equilibrium cannot necessarily be assumed. Also, we note several applications related to the inertial confinement fusion where under-dense plasma is considered and the effects of an anisotropic laser heating are studied [12].

When nonlocal thermodynamic equilibrium is assumed, several alternative approaches have been proposed. The first one, developed by Grad [15], considered a 13 moment closure but led to a system of equations which were not always hyperbolic. More recently, a new procedure was developed by Levermore [19] to generate a hierarchy of moment closure systems. The simplest model is the Euler equations with five equations. The second derived model, investigated by Levermore and Morokoff [20, admits 10 equations: the 10-moment Gaussian closure model.

The recent work of Dubroca et al. 12 uses this model to study the effect of the anisotropic phenomenon. Actually, the HLLE scheme [16 is used to approximate the weak solutions of the model. Since the numerical simulation of flows governed by this model are known to be difficult, the authors recommend a very robust scheme. The accuracy of the approximate solutions is lost with a large numerical

Received by the editor August 4, 2004 and, in revised form, September 13, 2005.

2000 Mathematics Subject Classification. Primary 65M99, 76N15; Secondary 76P05.

Key words and phrases. Hyperbolic system of conservation laws, Gaussian moment closure, relaxation scheme, discrete entropy inequalities. 
diffusion. In order to associate robustness and accuracy, we propose to develop a relaxation procedure.

Motivated by the work of Liu [22] and Chen, Levermore and Liu [9], the weak solutions of the 10-moment Gaussian closure are approximated by the weak solutions of a system which aims at restoring not only the initial model but also all its entropy inequalities. The main difference from the first relaxation scheme proposed by Jin and Xin [17] and studied by Natalini [23] and Aregba and Natalini [1] is that only the nonlinearities related to the pressure law are relaxed. These partial relaxation procedures have been introduced in distinct settings by Coquel and Perthame 11 and Coquel et al. 10. More recently, several extensions of the procedures have been developed by Baudin et al. [2] and Berthon et al. 4, 5] in distinct framework but for very difficult numerical simulations.

In the present work, we pay particular attention to the stability properties. In the framework of the usual $3 \times 3$ Euler equations, Bouchut [7] and Chalons and Coquel [8] study the discrete entropy inequalities using two distinct approaches. Related to the work of Chalons and Coquel [8, Berthon 3. proposes a third proof which finds a direct extension in the present framework of the 10-moment Gaussian closure model.

The derivation of the model and its main properties are presented in the next section. The third section is devoted to the numerical procedure. In the first step, we derive the relaxation model and establish its main properties. Next, we detail the numerical method. In the following section, we prove all the stability properties. Namely, we establish the positiveness of density and pressure, a set of discrete entropy inequalities, and also a maximum principle for the specific entropies. In the last section, the work is concluded by a numerical illustration of the scheme.

\section{The MATHEMATiCAL MODEL}

The flow under consideration is assumed to be governed by the 10-moment Gaussian closure for gas dynamics. This gas is characterized by its density $\rho>0$, its velocity $\mathbf{u} \in \mathbb{R}^{3}$, and its anisotropic total energy tensor $\mathbf{E} \in \mathbb{R}^{3} \times \mathbb{R}^{3}$. The system of PDE which governs such a flow is given by [20]:

$$
\left\{\begin{array}{l}
\partial_{t} \rho+\nabla \cdot(\rho \mathbf{u})=0, \\
\partial_{t}(\rho \mathbf{u})+\nabla \cdot(\rho \mathbf{u} \vee \mathbf{u}+\mathbf{p})=0, \\
\partial_{t} \mathbf{E}+\nabla \cdot((\mathbf{E}+\mathbf{p}) \vee \mathbf{u})=\Xi(\rho, \mathbf{p}),
\end{array}\right.
$$

where $\vee$ denotes the symmetric tensor outer product. The anisotropic pressure $\mathbf{p} \in \mathbb{R}^{3} \times \mathbb{R}^{3}$ satisfies the following tensorial state law:

$$
\mathbf{E}=\frac{1}{2} \rho \mathbf{u} \vee \mathbf{u}+\frac{1}{2} \mathbf{p}
$$

The pressure tensor is assumed to be a symmetric positive definite tensor.

The collisional term $\Xi$ will be systematically omitted in the sequel. Indeed, the present work concerns the numerical approximations of the weak solutions of the first order extracted system from (2.1); i.e., with a vanishing collisional term. This approach enters the usual strategy where an operator splitting is considered (for instance, see Dubroca et al. [12]). The present work is devoted to the first step of the splitting.

For the sake of simplicity in the forthcoming statements, we consider a bidimensional flow. As a consequence, we do not resolve the unknowns associated to 
the third direction and the system (2.1) reduces from 10 unknowns to 6 unknowns. Of course, all the results stated in the present paper easily extend to the full 3D model. In addition, arguing the rotational invariance of the system (2.1), we focus our attention on the single dimension problem which rewrites after a straightforward expansion of (2.1):

$$
\left\{\begin{array}{l}
\partial_{t} \rho+\partial_{x}\left(\rho u_{1}\right)=0, \quad t>0, x \in \mathbb{R} \\
\partial_{t}\left(\rho u_{1}\right)+\partial_{x}\left(\rho u_{1}^{2}+p_{11}\right)=0 \\
\partial_{t}\left(\rho u_{2}\right)+\partial_{x}\left(\rho u_{1} u_{2}+p_{12}\right)=0 \\
\partial_{t} E_{11}+\partial_{x}\left(\left(E_{11}+p_{11}\right) u_{1}\right)=0 \\
\partial_{t} E_{22}+\partial_{x}\left(E_{22} u_{1}+p_{12} u_{2}\right)=0 \\
\partial_{t} E_{12}+\partial_{x}\left(E_{12} u_{1}+\frac{1}{2}\left(p_{11} u_{2}+p_{12} u_{1}\right)\right)=0
\end{array}\right.
$$

where the state law (2.2) reads:

$$
E_{i j}=\frac{1}{2} \rho u_{i} u_{j}+\frac{1}{2} p_{i j}, \quad 1 \leq i \leq j \leq 2 .
$$

With clear notation, it will be convenient to write (2.3) under the following abstract form:

$$
\begin{aligned}
& \partial_{t} \mathbf{w}+\partial_{x} \mathbf{f}(\mathbf{w})=0, \\
& \mathbf{w}={ }^{t}\left(\rho, \rho u_{1}, \rho u_{2}, E_{11}, E_{22}, E_{12}\right) .
\end{aligned}
$$

We have $\mathbf{w}: \mathbb{R} \times \mathbb{R}_{+} \rightarrow \Omega$, where the state space $\Omega$ denotes the following open set:

$$
\Omega=\left\{\mathbf{w} \in \mathbb{R}^{6} ; \rho>0,\left(u_{1}, u_{2}\right) \in \mathbb{R}^{2}, p_{11}>0, p_{11} p_{22}-p_{12}^{2}>0\right\} .
$$

First, we state the following easy result, the proof of which is left to the reader (see also Gombosi et al. [13]):

Lemma 2.1. The system (2.3)-(2.4) is hyperbolic over $\Omega$ and admits the eigenvalues

$$
u_{1}, u_{1} \pm \sqrt{\frac{3 p_{11}}{\rho}}, u_{1} \pm \sqrt{\frac{p_{11}}{\rho}} .
$$

The eigenvalue $u_{1}$ has two orders of multiplicity, and it is associated with a linearly degenerated field. The other eigenvalues have one order of multiplicity. The eigenvalues $u_{1} \pm \sqrt{\frac{3 p_{11}}{\rho}}$ are associated with a genuinely nonlinear field while the last two are associated to a linearly degenerated field.

In addition to the above result, we establish several conservation equations satisfied by the smooth solutions of (2.3). These additional laws yield to entropy inequalities needed to rule out unphysical solutions.

Lemma 2.2. The smooth solutions $\mathbf{w} \in \Omega$ of (2.3) satisfy

$$
\begin{aligned}
& \partial_{t} \rho \mathcal{F}(s)+\partial_{x} \rho \mathcal{F}(s) u_{1}=0, \quad s:=s(\mathbf{w})=\frac{p_{11}}{\rho^{3}}, \\
& \partial_{t} \rho \mathcal{G}(\sigma)+\partial_{x} \rho \mathcal{G}(\sigma) u_{1}=0, \quad \sigma:=\sigma(\mathbf{w})=\frac{p_{11} p_{22}-p_{12}^{2}}{\rho^{4}},
\end{aligned}
$$

where $\mathcal{F}, \mathcal{G}: \mathbb{R} \rightarrow \mathbb{R}$ denote smooth functions. 
Assume

$$
\begin{aligned}
& \mathcal{F}=\tilde{\mathcal{F}} \circ \ln , \quad \tilde{\mathcal{F}}^{\prime}(y)<0, \quad \frac{\tilde{\mathcal{F}}^{\prime \prime}(y)}{\tilde{\mathcal{F}}^{\prime}(y)}<\frac{1}{3}, \quad \forall y \in \mathbb{R}, \\
& \mathcal{G}=\tilde{\mathcal{G}} \circ \ln , \quad \tilde{\mathcal{G}}^{\prime}(y)<0, \quad \frac{\tilde{\mathcal{G}}^{\prime \prime}(y)}{\tilde{\mathcal{G}}^{\prime}(y)}<\frac{1}{4}, \quad \forall y \in \mathbb{R} .
\end{aligned}
$$

Then both functions $\mathbf{w} \rightarrow \rho \mathcal{F}(s(\mathbf{w}))$ and $\mathbf{w} \rightarrow \rho \mathcal{G}(\sigma(\mathbf{w}))$ are convex. As a consequence, the pairs $\left(\rho \mathcal{F}(s), \rho \mathcal{F}(s) u_{1}\right)$ and $\left(\rho \mathcal{G}(\sigma), \rho \mathcal{G}(\sigma) u_{1}\right)$ define Lax entropy pairs for the system (2.3):

$$
\partial_{t} \rho \mathcal{F}(s)+\partial_{x} \rho \mathcal{F}(s) u_{1} \leq 0 \quad \text { and } \quad \partial_{t} \rho \mathcal{G}(\sigma)+\partial_{x} \rho \mathcal{G}(\sigma) u_{1} \leq 0 .
$$

Proof. As soon as the solution of (2.3) is smooth enough, both momentum equations can be developed to obtain

$$
\begin{aligned}
& \partial_{t} u_{1}+u_{1} \partial_{x} u_{1}+\frac{1}{\rho} \partial_{x} p_{11}=0 \\
& \partial_{t} u_{2}+u_{1} \partial_{x} u_{2}+\frac{1}{\rho} \partial_{x} p_{12}=0
\end{aligned}
$$

so that we deduce

$$
\begin{aligned}
& \partial_{t} \rho \frac{u_{1}^{2}}{2}+\partial_{x} \rho \frac{u_{1}^{2}}{2} u_{1}+u_{1} \partial_{x} p_{11}=0, \\
& \partial_{t} \rho \frac{u_{2}^{2}}{2}+\partial_{x} \rho \frac{u_{2}^{2}}{2} u_{1}+u_{2} \partial_{x} p_{12}=0, \\
& \partial_{t} \rho \frac{u_{1} u_{2}}{2}+\partial_{x} \rho \frac{u_{1} u_{2}}{2} u_{1}+\frac{1}{2}\left(u_{2} \partial_{x} p_{11}+u_{1} \partial_{x} p_{12}\right)=0 .
\end{aligned}
$$

Now, we subtract each kinetic energy $\rho \frac{u_{i} u_{j}}{2}$ for $1 \leq i \leq j \leq 2$ from the associated total energy $E_{i j}$ to obtain after computations:

$$
\begin{aligned}
& \partial_{t} p_{11}+u_{1} \partial_{x} p_{11}+3 p_{11} \partial_{x} u_{1}=0 \\
& \partial_{t} p_{22}+u_{1} \partial_{x} p_{22}+p_{22} \partial_{x} u_{1}+2 p_{12} \partial_{x} u_{2}=0 \\
& \partial_{t} p_{12}+u_{1} \partial_{x} p_{12}+p_{11} \partial_{x} u_{2}+2 p_{12} \partial_{x} u_{1}=0
\end{aligned}
$$

Since

$$
\partial_{t} \rho+u_{1} \partial_{x} \rho+\rho \partial_{x} u_{1}=0
$$

we have

$$
\frac{1}{\rho^{3}}\left(\partial_{t} p_{11}+u_{1} \partial_{x} p_{11}+3 p_{11} \partial_{x} u_{1}\right)-\frac{3 p_{11}}{\rho^{4}}\left(\partial_{t} \rho+u_{1} \partial_{x} \rho+\rho \partial_{x} u_{1}\right)=0
$$

which reads

$$
\partial_{t} s+u_{1} \partial_{x} s=0 \quad \text { with } \quad s=\frac{p_{11}}{\rho^{3}} .
$$

Now, for all $\mathcal{F} \in C^{1}(\mathbb{R})$ we write

$$
\partial_{t} \mathcal{F}(s)+u_{1} \partial_{x} \mathcal{F}(s)=0 .
$$


Since we have

$$
\rho\left(\partial_{t} \mathcal{F}(s)+u_{1} \partial_{x} \mathcal{F}(s)\right)+s\left(\partial_{t} \rho+\partial_{x} \rho u_{1}\right)=\partial_{t} \rho \mathcal{F}(s)+\partial_{x} \rho \mathcal{F}(s) u_{1},
$$

the relation (2.6) is established. Concerning the identity (2.7), a straightforward computation gives

$$
\partial_{t}\left(p_{11} p_{22}-p_{12}^{2}\right)+u_{1} \partial_{x}\left(p_{11} p_{22}-p_{12}^{2}\right)+4\left(p_{11} p_{22}-p_{12}^{2}\right) \partial_{x} u_{1}=0
$$

Then we easily deduce

$$
\partial_{t} \frac{p_{11} p_{22}-p_{12}^{2}}{\rho^{4}}+u_{1} \partial_{x} \frac{p_{11} p_{22}-p_{12}^{2}}{\rho^{4}}=0,
$$

and the proof of (2.7) is concluded similarly to the proof of (2.6).

Concerning the convexity result, as usual the proof is obtained when studying the Hessian matrix of the functions $\mathbf{w} \rightarrow \rho \mathcal{F}(s(\mathbf{w}))$ and $\mathbf{w} \rightarrow \rho \mathcal{G}(\sigma(\mathbf{w}))$ (see also Godlewski and Raviart [14]).

To summarize our problem, we consider a conservative hyperbolic system (2.3) completed by the entropy inequalities (2.10). Moreover, the solutions of this problem belong to the admissible state space $\Omega$. Our goal is to propose a numerical procedure to approximate the solutions defined in $\Omega$ of (2.3)-(2.4) supplemented by (2.10). To access such an issue, we will develop a relaxation scheme.

\section{A Relaxation method}

We propose to approximate the weak solutions of the system (2.3)-(2.10) by the weak solutions of a relevant first order system with singular perturbations: the relaxation model. Several works are devoted to such approaches (for instance, see Liu 22] or Chen, Levermore and Liu [9]), where the relaxation model aims to restore the initial model completed by the entropy inequalities within the limit of an infinite relaxation parameter.

Motivated by the work of Coquel and Perthame [11, Chalons and Coquel [8], and Baudin et al. 22, we propose a relaxation model which preserves most of the nonlinearities of the initial system. These nonlinearities are kept in order to enforce the contact waves associated to the eigenvalue $u_{1}$ of (2.3) to be solutions of our relaxation model. In this sense, the present paper differs from the pioneering work of Jin and Xin [17, where all the nonlinearities are relaxed.

3.1. The relaxation model. Following the work of Suliciu [25] (see also [2, 8, 11]), we propose a relevant modification of the pressure law. We suggest substituting the pressures $p_{11}$ and $p_{12}$ by the approximation $\pi_{11}$ and $\pi_{12}$, where these two new variables are governed by

$$
\left\{\begin{array}{l}
\partial_{t} \pi_{11}+u_{1} \partial_{x} \pi_{11}+\frac{a^{2}}{\rho} \partial_{x} u_{1}=\lambda\left(p_{11}-\pi_{11}\right), \\
\partial_{t} \pi_{12}+u_{1} \partial_{x} \pi_{12}+\frac{a^{2}}{\rho} \partial_{x} u_{2}=\lambda\left(p_{12}-\pi_{12}\right) .
\end{array}\right.
$$

The relaxation parameter $a$ must satisfy an additional stability condition, the wellknown sub-characteristic Whitham condition [26], detailed later on. 
Thus, we approximate the entropy weak solutions of (2.3)-(2.4)-(2.10) by those of the following first order system with singular perturbations:

$$
\left\{\begin{array}{l}
\partial_{t} \rho+\partial_{x}\left(\rho u_{1}\right)=0, \quad t>0, x \in \mathbb{R} \\
\partial_{t}\left(\rho u_{1}\right)+\partial_{x}\left(\rho u_{1}^{2}+\pi_{11}\right)=0 \\
\partial_{t}\left(\rho u_{2}\right)+\partial_{x}\left(\rho u_{1} u_{2}+\pi_{12}\right)=0 \\
\partial_{t} E_{11}+\partial_{x}\left(\left(E_{11}+\pi_{11}\right) u_{1}\right)=0 \\
\partial_{t} E_{22}+\partial_{x}\left(E_{22} u_{1}+\pi_{12} u_{2}\right)=0 \\
\partial_{t} E_{12}+\partial_{x}\left(E_{12} u_{1}+\frac{1}{2}\left(\pi_{11} u_{2}+\pi_{12} u_{1}\right)\right)=0 \\
\partial_{t} \rho \pi_{11}+\partial_{x}\left(\rho \pi_{11} u_{1}+a^{2} u_{1}\right)=\lambda \rho\left(p_{11}-\pi_{11}\right) \\
\partial_{t} \rho \pi_{12}+\partial_{x}\left(\rho \pi_{12} u_{1}+a^{2} u_{2}\right)=\lambda \rho\left(p_{12}-\pi_{12}\right)
\end{array}\right.
$$

As soon as the relaxation parameter $\lambda$ goes to infinity, from (3.1) we formally recover the initial system (2.3). Indeed, in this limit referred to as the equilibrium limit, we have $\pi_{11}=p_{11}$ and $\pi_{12}=p_{12}$, and thus the conservation of the momentum $\left(\rho u_{1}\right.$ and $\left.\rho u_{2}\right)$ and the total energy $\left(E_{11}, E_{22}\right.$ and $\left.E_{12}\right)$ in (3.1) gives those of (2.3).

Let us note from now on that according to the work of Liu [22] and Chen, and Levermore and Liu 9, both systems (2.3) and (3.1) must satisfy compatibility conditions to prevent instabilities in the regime of infinite $\lambda$. These so-called subcharacteristic Whitham conditions read as follows:

$$
\frac{a^{2}}{\rho}>3 p_{11} \text {. }
$$

We will see that this condition enters in the analysis of the discrete entropy inequalities.

With clear notation, let us introduce the following abstract form of the relaxation system (3.1):

$$
\left\{\begin{array}{l}
\partial_{t} \mathbf{W}+\partial_{x} \mathbf{F}_{a}(\mathbf{W})=\lambda \mathbf{R}(\mathbf{W}), \\
\mathbf{W}={ }^{t}\left(\rho, \rho u_{1}, \rho u_{2}, E_{11}, E_{22}, E_{12}, \rho \pi_{11}, \rho \pi_{12}\right),
\end{array}\right.
$$

associated with the phase space

$$
\mathcal{V}=\left\{\mathbf{W} \in \mathbb{R}^{8} ; \rho>0\right\} .
$$

In the flux function notation, we have introduced the subscript $a$ to emphasize the dependence on the relaxation parameter $a$.

In fact, the derivation of the relaxation model (3.1) is dictated by a linear degeneracy of all the fields, which makes it an easily solvable Riemann problem. The first statement we give is devoted to the linear degeneracy property of the fields.

Lemma 3.1. Let be given $a>0$ and assume $\lambda=0$. The first order system (3.1) is hyperbolic for all $\mathbf{W} \in \mathcal{V}$. It admits $\mu_{1}=u_{1}-a / \rho$ and $\mu_{3}=u_{1}+a / \rho$ as double eigenvalues and $\mu_{2}=u_{1}$ with a multiplicity four. All the fields are linearly degenerated.

We do not give the proof of this result which turns out to be easy and usual (see Godlewski and Raviart [14]). In the next statement, we give the solution of the Riemann problem for the relaxation system (3.1) with $\lambda=0$. Since all the fields are linearly degenerate, the Riemann solution is made of four constant states separated by three contact discontinuities. 
Lemma 3.2. Let $\mathbf{W}_{L}$ and $\mathbf{W}_{R}$ be constant states in $\mathcal{V}$ and define

$$
\mathbf{W}_{0}(x)= \begin{cases}\mathbf{W}_{L} & \text { if } x<0, \\ \mathbf{W}_{R} & \text { if } x>0,\end{cases}
$$

the initial data for the system (3.1) with $\lambda=0$. Assume that the relaxation constant a satisfies

$$
\begin{aligned}
& \mu_{1}\left(\mathbf{W}_{L}\right)=\left(u_{1}\right)_{L}-\frac{a}{\rho_{L}}<u_{1}^{\star}<\mu_{3}\left(\mathbf{W}_{R}\right)=\left(u_{1}\right)_{R}+\frac{a}{\rho_{R}}, \\
& u_{1}^{\star}=\frac{\left(u_{1}\right)_{L}+\left(u_{1}\right)_{R}}{2}+\frac{\left(\pi_{11}\right)_{L}-\left(\pi_{11}\right)_{R}}{2 a} .
\end{aligned}
$$

Then the weak solution of the system (3.1) with $\lambda=0$ and for the initial data (3.4) is given by

$$
\mathbf{W}(x, t)=\left\{\begin{array}{cllll}
\mathbf{W}_{L} & \text { if } & & \frac{x}{t}<\mu_{1}\left(\mathbf{W}_{L}\right), \\
\mathbf{W}_{1} & \text { if } & \mu_{1}\left(\mathbf{W}_{L}\right)< & \frac{x}{t}<\mu_{2}\left(\mathbf{W}_{1}\right), \\
\mathbf{W}_{2} & \text { if } \mu_{2}\left(\mathbf{W}_{2}\right)< & \frac{x}{t} & <\mu_{3}\left(\mathbf{W}_{R}\right), \\
\mathbf{W}_{R} & \text { if } \quad \mu_{3}\left(\mathbf{W}_{R}\right)<\frac{x}{t}, &
\end{array}\right.
$$

where $\mu_{2}\left(\mathbf{W}_{1}\right)=\mu_{2}\left(\mathbf{W}_{2}\right)=u_{1}^{\star}$. Let us set for all $1 \leq i \leq j \leq 2$

$$
\begin{aligned}
u_{i}^{\star} & =\frac{\left(u_{i}\right)_{L}+\left(u_{i}\right)_{R}}{2}+\frac{\left(\pi_{1 i}\right)_{L}-\left(\pi_{1 i}\right)_{R}}{2 a}, \\
\pi_{1 i}^{\star} & =\frac{\left(\pi_{1 i}\right)_{L}+\left(\pi_{1 i}\right)_{R}}{2}+\frac{a}{2}\left(\left(u_{i}\right)_{L}-\left(u_{i}\right)_{R}\right), \\
\frac{1}{\rho^{1}} & =\frac{1}{\rho_{L}}+\frac{u_{1}^{\star}-\left(u_{1}\right)_{L}}{a}, \frac{1}{\rho^{2}}=\frac{1}{\rho_{R}}+\frac{\left(u_{1}\right)_{R}-u_{1}^{\star}}{a}, \\
e_{i j}^{1} & =\left(\frac{\left(E_{i j}\right)_{L}}{\rho_{L}}-\frac{\left(u_{i}\right)_{L}\left(u_{j}\right)_{L}}{2}\right)-\frac{1}{2 a^{2}}\left(\left(\pi_{1 i}\right)_{L}\left(\pi_{1 j}\right)_{L}-\pi_{1 i}^{\star} \pi_{1 j}^{\star}\right), \\
e_{i j}^{2} & =\left(\frac{\left(E_{i j}\right)_{R}}{\rho_{R}}-\frac{\left(u_{i}\right)_{R}\left(u_{j}\right)_{R}}{2}\right)-\frac{1}{2 a^{2}}\left(\left(\pi_{1 i}\right)_{R}\left(\pi_{1 j}\right)_{R}-\pi_{1 i}^{\star} \pi_{1 j}^{\star}\right), \\
E_{i j}^{1} & =\rho^{1} \frac{u_{i}^{\star} u_{j}^{\star}}{2}+\rho^{1} e_{i j}^{1}, \quad E_{i j}^{2}=\rho^{2} \frac{u_{i}^{\star} u_{j}^{\star}}{2}+\rho^{2} e_{i j}^{2} .
\end{aligned}
$$

Then the constant states $\mathbf{W}_{1}$ and $\mathbf{W}_{2}$ in $\mathcal{V}$ are defined as follows:

$$
\mathbf{W}_{i}={ }^{t}\left(\rho^{i}, \rho^{i} u_{1}^{\star}, \rho^{i} u_{2}^{\star}, E_{11}^{i}, E_{22}^{i}, E_{12}^{i}, \rho^{i} \pi_{11}^{\star}, \rho^{i} \pi_{12}^{\star}\right), \quad 1 \leq i \leq 2 .
$$

Proof. Since all the fields are linearly degenerate, the Riemann solution is made of contact discontinuities. The $i$ th discontinuity propagates the velocity $\mu_{i}$. These discontinuities separate constant states: $\mathbf{W}_{0}=\mathbf{W}_{L}, \mathbf{W}_{1}, \mathbf{W}_{2}, \mathbf{W}_{3}=\mathbf{W}_{R}$. Two consecutive states satisfy the Rankine-Hugoniot jump conditions associated with the system (3.3) with $\lambda=0$ :

$$
-\mu_{i}\left(\mathbf{W}_{i}\right)\left(\mathbf{W}_{i}-\mathbf{W}_{i-1}\right)+\left(\mathbf{F}_{a}\left(\mathbf{W}_{i}\right)-\mathbf{F}_{a}\left(\mathbf{W}_{i-1}\right)\right)=0,
$$

with $1 \leq i \leq 3$. Since the coefficient $a$ satisfies (3.5), which coincides with the ordering of the three waves, the relations (3.6) easily yield the definition of the intermediate states $\mathbf{W}_{1}$ and $\mathbf{W}_{2}$.

To conclude the proof, we establish the positiveness of $\rho^{1}$ and $\rho^{2}$ which is a direct consequence of (3.5). Indeed, the linear degeneracy of each field implies $\mu_{1}\left(\mathbf{W}_{L}\right)=\mu_{1}\left(\mathbf{W}_{1}\right)$ and $\mu_{3}\left(\mathbf{W}_{R}\right)=\mu_{3}\left(\mathbf{W}_{2}\right)$. Then, the condition (3.5) rewrites $\rho^{1}>0$ and $\rho^{2}>0$. 
To conclude the presentation of the relaxation model, we propose to perform a small perturbation analysis to give the first-order asymptotic system. This derivation is obtained by the Chapman-Enskog procedure (for instance, see Chen, Levermore and Liu [9], Coquel and Perthame [11, or Liu [22]). To access such an issue, let us consider the formal expansion of $\pi_{11}$ and $\pi_{12}$ in the form

$$
\begin{aligned}
& \pi_{11}=p_{11}+\frac{1}{\lambda} \pi_{11}^{1}+O\left(\frac{1}{\lambda^{2}}\right), \\
& \pi_{12}=p_{12}+\frac{1}{\lambda} \pi_{12}^{1}+O\left(\frac{1}{\lambda^{2}}\right) .
\end{aligned}
$$

From the evolution laws of $\rho \pi_{11}$ and $\rho \pi_{12}$ in (3.1), we obtain the following first-order correction:

$$
\begin{aligned}
& \pi_{11}^{1}=-\left(\frac{a^{2}}{\rho}-3 p_{11}\right) \partial_{x} u_{1} \\
& \pi_{12}^{1}=-2 p_{12} \partial_{x} u_{1}-\left(\frac{a^{2}}{\rho}-p_{11}\right) \partial_{x} u_{2}
\end{aligned}
$$

Equipped with these expansions, we deduce that the first-order asymptotic equilibrium system reads:

$$
\left\{\begin{aligned}
\partial_{t} \rho+\partial_{x}\left(\rho u_{1}\right)=0 \\
\partial_{t}\left(\rho u_{1}\right)+\partial_{x}\left(\rho u_{1}^{2}+p_{11}\right)=\frac{1}{\lambda} \partial_{x}\left(\nu_{1} \partial_{x} u_{1}\right) \\
\partial_{t}\left(\rho u_{2}\right)+\partial_{x}\left(\rho u_{1} u_{2}+p_{12}\right)=\frac{1}{\lambda} \partial_{x}\left(2 p_{12} \partial_{x} u_{1}+\nu_{2} \partial_{x} u_{2}\right) \\
\partial_{t} E_{11}+\partial_{x}\left(\left(E_{11}+p_{11}\right) u_{1}\right)=\frac{1}{\lambda} \partial_{x}\left(\nu_{1} u_{1} \partial_{x} u_{1}\right) \\
\partial_{t} E_{22}+\partial_{x}\left(E_{22} u_{1}+p_{12} u_{2}\right)=\frac{1}{\lambda} \partial_{x}\left(2 p_{12} u_{1} \partial_{x} u_{1}+\nu_{2} u_{2} \partial_{x} u_{2}\right) \\
\partial_{t} E_{12}+\partial_{x}\left(E_{12} u_{1}+\frac{1}{2}\left(p_{11} u_{2}+p_{12} u_{1}\right)\right) \\
=\frac{1}{2 \lambda} \partial_{x}\left(\nu_{1} u_{2} \partial_{x} u_{1}+\nu_{2} u_{1} \partial_{x} u_{2}+2 p_{12} u_{1} \partial_{x} u_{1}\right)
\end{aligned}\right.
$$

where we have set

$$
\nu_{1}=\frac{a^{2}}{\rho}-3 p_{11} \quad \text { and } \quad \nu_{2}=\frac{a^{2}}{\rho}-p_{11} .
$$

To complete the first-order asymptotic behaviors, we have to consider the full system with the collisional terms $\Xi$. In [20, Levermore and Morokoff prescribe the following closure:

$$
\Xi=\lambda(p \mathbf{I}-\mathbf{p}), \quad p=\alpha p_{11}+(1-\alpha) p_{22},
$$

where $\alpha \in(0,1)$. In fact, the collisional term is nothing but a relaxation source term which relaxes $p_{11}$ to $p$ and $p_{12}$ to zero. For the sake of clarity in this brief presentation, we consider the $1 \mathrm{D}$ problem: the velocity $u_{2}$ in the orthogonal direction is set to zero. We introduce the following total energy:

$$
E=E_{11}+\frac{1-\alpha}{\alpha} E_{22}
$$


Using the Chapman-Enskog expansion, the relaxation model (3.1) with the collisional term gives the following formal first-order asymptotic equilibrium system:

$$
\left\{\begin{array}{l}
\partial_{t} \rho+\partial_{x}\left(\rho u_{1}\right)=0 \\
\partial_{t}\left(\rho u_{1}\right)+\partial_{x}\left(\rho u_{1}^{2}+p\right)=\frac{1}{\lambda} \partial_{x}\left(\mu_{1} \partial_{x} u_{1}\right), \\
\partial_{t} E+\partial_{x}\left((E+p) u_{1}\right)=\frac{1}{\lambda} \partial_{x}\left(\mu_{1} u_{1} \partial_{x} u_{1}\right) .
\end{array}\right.
$$

The state law satisfied by the pressure reads

$$
p=(\gamma-1)\left(E-\rho \frac{u_{1}^{2}}{2}\right), \quad \gamma \in(1,3),
$$

where we have set $\gamma=1+2 \alpha$.

In the system (3.8), we recognize the Navier-Stokes equations where the viscosity function is given by

$$
\mu_{1}=\frac{a^{2}}{\rho}-\gamma p,
$$

which defines a positive function as soon as the Whitham condition (3.2) is assumed.

3.2. The relaxation scheme. On the basis of the above relaxation model (3.1), we propose to describe the numerical procedure which turns out to be usual in the frame work of the relaxation scheme (see Jin and Xin [17, Coquel and Perthame [11, Baudin et al. [2]).

We consider a structured mesh in space and time defined by the cells $I_{i}=$ $\left[x_{i-\frac{1}{2}}, x_{i+\frac{1}{2}}\right)$ and the time intervals $\left[t^{n}, t^{n+1}\right)$ :

$$
t^{n}=n \Delta t \quad \text { and } \quad x_{i+\frac{1}{2}}=\left(i+\frac{1}{2}\right) \Delta x \quad \text { with } \quad i \in \mathbb{Z}, n \in \mathbb{N},
$$

where $\Delta t$ is the time increment and $\Delta x$ the spatial cell width.

As usual, we assume that a piecewise constant approximate equilibrium solution $\mathbf{w}^{h}\left(x, t^{n}\right) \in \Omega$ is known at time $t^{n}$, defined by

$$
\mathbf{w}^{h}\left(x, t^{n}\right)=\mathbf{w}_{i}^{n}={ }^{t}\left(\rho_{i}^{n},\left(\rho u_{1}\right)_{i}^{n},\left(\rho u_{2}\right)_{i}^{n},\left(E_{11}\right)_{i}^{n},\left(E_{22}\right)_{i}^{n},\left(E_{12}\right)_{i}^{n}\right), \quad x \in I_{i} .
$$

At the initial time, we set

$$
\mathbf{w}_{i}^{0}=\frac{1}{\Delta x} \int_{x_{i-\frac{1}{2}}}^{x_{i+\frac{1}{2}}} \mathbf{w}(x, 0) d x .
$$

The approximate equilibrium solution is then evolved to the next time level $t^{n+1}=$ $t^{n}+\Delta t$ by taking into account two steps.

First step: Evolution in time. We introduce $\mathbf{W}^{h} \in \mathcal{V}$ such that for all $0<t<\Delta t$, the function $\mathbf{W}^{h}\left(x, t^{n}+t\right)$ is the weak solution of the Cauchy problem for the relaxation system (3.1) with $\lambda=0$ :

$$
\partial_{t} \mathbf{W}+\partial_{x} \mathbf{F}_{a}(\mathbf{W})=0,
$$

for the following initial equilibrium data:

$$
\begin{aligned}
& \mathbf{W}^{h}\left(x, t^{n}\right)=\mathbf{W}_{i}^{n} \\
& \quad={ }^{t}\left(\rho_{i}^{n},\left(\rho u_{1}\right)_{i}^{n},\left(\rho u_{2}\right)_{i}^{n},\left(E_{11}\right)_{i}^{n},\left(E_{22}\right)_{i}^{n},\left(E_{12}\right)_{i}^{n},\left(\rho \pi_{11}\right)_{i}^{n},\left(\rho \pi_{12}\right)_{i}^{n}\right), \quad x \in I_{i},
\end{aligned}
$$


where $\left(\pi_{11}\right)_{i}^{n}=\left(p_{11}\right)_{i}^{n}$ and $\left(\pi_{12}\right)_{i}^{n}=\left(p_{12}\right)_{i}^{n}$. With some abuse in the notation, we have set

$$
\left(p_{l k}\right)_{i}^{n}=2\left(\left(E_{l k}\right)_{i}^{n}-\frac{\left(\rho u_{k}\right)_{i}^{n}\left(\rho u_{l}\right)_{i}^{n}}{2 \rho_{i}^{n}}\right) .
$$

Under the CFL-like condition

$$
\frac{\Delta t}{\Delta x} \max _{i \in \mathbb{Z}}\left(\left|\mu_{1}\left(\mathbf{W}_{i}^{n}\right)\right|,\left|\mu_{3}\left(\mathbf{W}_{i}^{n}\right)\right|\right) \leq \frac{1}{2},
$$

the solution $\mathbf{W}^{h}$ at the time $t^{n}+\Delta t$ is made of the juxtaposition of the noninteracting Riemann problem solution set at the cell interfaces $x_{i+\frac{1}{2}}$ for $i \in \mathbb{Z}$.

Next, the projection of this solution on the piecewise constant functions gives

$$
\mathbf{W}_{i}^{n+1,-}=\frac{1}{\Delta x} \int_{x_{i-\frac{1}{2}}}^{x_{i+\frac{1}{2}}} \mathbf{W}^{h}\left(x, t^{n}+\Delta t\right) d x .
$$

In fact, a local definition of the parameter $a$ at each interface $x_{i+\frac{1}{2}}$ can be considered (for instance, see Coquel and Perthame [11] or Baudin et al. 2]). At each interface $x_{i+\frac{1}{2}}$, we choose $\mathbf{W}_{L}=\mathbf{W}_{i}^{n}$ and $\mathbf{W}_{R}=\mathbf{W}_{i+1}^{n}$ to define the parameter $a_{i+\frac{1}{2}}$ according to the Whitham condition (3.2) and the ordering condition (3.5). Assuming the CFL restriction (3.10), the relaxation parameter a may vary from one interface to another. For convenience in the sequel and to emphasize the admissible local choice of the parameter $a$, we rewrite $\mathbf{W}_{i}^{n+1,-}$ arguing the formalism introduced by Harten, Lax, and van Leer [16]:

$$
\mathbf{W}_{i}^{n+1,-}=\frac{1}{2}\left(\overline{\mathbf{W}}_{R}\left(\mathbf{W}_{i-1}^{n}, \mathbf{W}_{i}^{n}\right)+\overline{\mathbf{W}}_{L}\left(\mathbf{W}_{i}^{n}, \mathbf{W}_{i+1}^{n}\right)\right),
$$

where

$$
\begin{aligned}
\overline{\mathbf{W}}_{L}\left(\mathbf{W}_{L}, \mathbf{W}_{R}\right) & =\frac{2 \Delta t}{\Delta x} \int_{-\frac{\Delta x}{2 \Delta t}}^{0} \mathbf{W}_{a}\left(\xi ; \mathbf{W}_{L}, \mathbf{W}_{R}\right) d \xi \\
& =\mathbf{W}_{L}-\frac{2 \Delta t}{\Delta x}\left(\mathbf{F}_{a}\left(\mathbf{W}_{a}\left(0 ; \mathbf{W}_{L}, \mathbf{W}_{R}\right)\right)-\mathbf{F}_{a}\left(\mathbf{W}_{L}\right)\right)
\end{aligned}
$$

and

$$
\begin{aligned}
\overline{\mathbf{W}}_{R}\left(\mathbf{W}_{L}, \mathbf{W}_{R}\right) & =\frac{2 \Delta t}{\Delta x} \int_{0}^{\frac{\Delta x}{2 \Delta t}} \mathbf{W}_{a}\left(\xi ; \mathbf{W}_{L}, \mathbf{W}_{R}\right) d \xi \\
& =\mathbf{W}_{R}-\frac{2 \Delta t}{\Delta x}\left(\mathbf{F}_{a}\left(\mathbf{W}_{R}\right)-\mathbf{F}_{a}\left(\mathbf{W}_{a}\left(0 ; \mathbf{W}_{L}, \mathbf{W}_{R}\right)\right)\right) .
\end{aligned}
$$

The function $\mathbf{W}_{a}\left(. ; \mathbf{W}_{L}, \mathbf{W}_{R}\right)$ denotes the solution of the Riemann problem for (3.9) where the initial data is prescribed by (3.4). The index $a$ has been introduced to emphasize the dependence on the relaxation coefficient $a$.

As soon as $\mathbf{W}_{L}$ and $\mathbf{W}_{R}$ are defined from the equilibrium states $\mathbf{w}_{L}$ and $\mathbf{w}_{R}$, it is crucial to note from now on the following identities:

$$
\begin{aligned}
& \left.\mathbf{F}_{a}\left(\mathbf{W}_{L}\right)\right|_{\left[\rho, \rho u_{1}, \rho u_{2}, E_{11}, E_{22}, E_{12}\right]}=f\left(\mathbf{w}_{L}\right), \\
& \left.\mathbf{F}_{a}\left(\mathbf{W}_{R}\right)\right|_{\left[\rho, \rho u_{1}, \rho u_{2}, E_{11}, E_{22}, E_{12}\right]}=f\left(\mathbf{w}_{R}\right),
\end{aligned}
$$

where the notation $\left.\mathbf{F}_{a}()\right|_{.\left[\rho, \rho u_{1}, \rho u_{2}, E_{11}, E_{22}, E_{12}\right]}$ denotes the restriction of $\mathbf{F}_{a}$ to the component $\left(\rho, \rho u_{1}, \rho u_{2}, E_{11}, E_{22}, E_{12}\right)$. 
Second step: The relaxation. At time $t=t^{n}+\Delta t$, we define the updated approximate equilibrium solution $\mathbf{w}^{n+1}(x)$ as follows:

$$
\begin{aligned}
\mathbf{w}^{n+1}(x)={ }^{t} & \left(\rho_{i}^{n+1,-},\left(\rho u_{1}\right)_{i}^{n+1,-},\left(\rho u_{2}\right)_{i}^{n+1,-},\right. \\
& \left.\left(E_{11}\right)_{i}^{n+1,-},\left(E_{22}\right)_{i}^{n+1,-},\left(E_{12}\right)_{i}^{n+1,-}\right), \quad x \in I_{i},
\end{aligned}
$$

and we set $\left(\pi_{11}\right)_{i}^{n+1}=\left(p_{11}\right)_{i}^{n+1}$ and $\left(\pi_{12}\right)_{i}^{n+1}=\left(p_{12}\right)_{i}^{n+1}$.

In fact, this second step amounts to solving the system

$$
\partial_{t} \mathbf{W}=\lambda \mathbf{R}(\mathbf{W})
$$

with the piecewise constant approximation $\mathbf{W}_{i}^{n+1,-}$ as initial data while $\lambda$ tends to infinity.

A summary of the scheme. To summarize, the numerical procedure we just described enters the classical framework of the usual finite volume methods exactly:

$$
\mathbf{w}_{i}^{n+1}=\mathbf{w}_{i}^{n}-\frac{\Delta t}{\Delta x}\left(\mathbf{f}_{i+\frac{1}{2}}^{n}-\mathbf{f}_{i-\frac{1}{2}}^{n}\right),
$$

where the numerical flux function is defined by

$$
\begin{aligned}
\mathbf{f}_{i+\frac{1}{2}}^{n} & =\mathbf{f}\left(\mathbf{w}_{i}^{n}, \mathbf{w}_{i+1}^{n}\right) \\
& =\left.\mathbf{F}_{a_{i+\frac{1}{2}}}\left(\mathbf{W}_{a_{i+\frac{1}{2}}}\left(0 ; \mathbf{W}\left(\mathbf{w}_{i}^{n}\right), \mathbf{W}\left(\mathbf{w}_{i+1}^{n}\right)\right)\right)\right|_{\left[\rho, \rho u_{1}, \rho u_{2}, E_{11}, E_{22}, E_{12}\right]},
\end{aligned}
$$

with $\mathbf{W}_{i}^{n}=\mathbf{W}\left(\mathbf{w}_{i}^{n}\right)$ defined according to the equilibrium, i.e., $\left(\pi_{11}\right)_{i}^{n}=\left(p_{11}\right)_{i}^{n}$ and $\left(\pi_{12}\right)_{i}^{n}=\left(p_{12}\right)_{i}^{n}$.

\section{The ENTROPy INEQUALITIES}

The present section is devoted to establishing all the required stability properties satisfied by the scheme (3.15)-(3.16). Namely, we establish the discrete formulation of all the entropy inequalities (2.10). In addition, we prove that the updated approximate solution $\mathbf{w}_{i}^{n+1}$ belongs to the admissible state space $\Omega$ as soon as $\mathbf{w}_{i}^{n} \in \Omega$. All these expected properties are obtained when assuming the CFL-like condition (3.10), the Whitham condition (3.2), and the ordering condition (3.5). No addition restrictions are assumed to establish the stability result. Now, we state our main result:

Theorem 4.1. Consider the relaxation scheme (3.15)-(3.16) and assume the CFLlike restriction (3.10), the Whitham sub-characteristic condition (3.2), and the ordering condition (3.5). Assume that $\mathbf{w}_{i}^{n} \in \Omega$ for all $i \in \mathbb{Z}$. Then the updated approximation $\mathbf{w}_{i}^{n+1}$ belongs to $\Omega$ for all $i \in \mathbb{Z}$ :

$$
\rho_{i}^{n+1}>0, \quad\left(p_{11}\right)_{i}^{n+1}>0, \quad\left(p_{11}\right)_{i}^{n+1}\left(p_{22}\right)_{i}^{n+1}-\left(\left(p_{12}\right)_{i}^{n+1}\right)^{2}>0 .
$$

In addition, the following discrete entropy inequalities are satisfied for all $i \in \mathbb{Z}$ :

$$
\begin{aligned}
& \rho_{i}^{n+1} \mathcal{F}\left(s_{i}^{n+1}\right)-\rho_{i}^{n} \mathcal{F}\left(s_{i}^{n}\right)+\frac{\Delta t}{\Delta x}\left(\left\{\rho \mathcal{F}(s) u_{1}\right\}_{i+\frac{1}{2}}^{n}-\left\{\rho \mathcal{F}(s) u_{1}\right\}_{i-\frac{1}{2}}^{n}\right) \leq 0, \\
& \rho_{i}^{n+1} \mathcal{G}\left(\sigma_{i}^{n+1}\right)-\rho_{i}^{n} \mathcal{G}\left(\sigma_{i}^{n}\right)+\frac{\Delta t}{\Delta x}\left(\left\{\rho \mathcal{G}(\sigma) u_{1}\right\}_{i+\frac{1}{2}}^{n}-\left\{\rho \mathcal{G}(\sigma) u_{1}\right\}_{i-\frac{1}{2}}^{n}\right) \leq 0,
\end{aligned}
$$


where

$$
s_{i}^{n}=\frac{\left(p_{11}\right)_{i}^{n}}{\left(\rho_{i}^{n}\right)^{3}}, \quad \sigma_{i}^{n}=\frac{\left(p_{11}\right)_{i}^{n}\left(p_{22}\right)_{i}^{n}-\left(\left(p_{12}\right)_{i}^{n}\right)^{2}}{\left(\rho_{i}^{n}\right)^{4}} .
$$

The functions $\mathcal{F}$ and $\mathcal{G}$ satisfy the convexity assumptions (2.8) and (2.9), respectively. The discrete entropy numerical flux functions are defined as follows:

$$
\begin{aligned}
& \left\{\rho \mathcal{F}(s) u_{1}\right\}_{i+\frac{1}{2}}^{n}=\left(\mathbf{f}_{\rho}\right)_{i+\frac{1}{2}}^{n} \times \begin{cases}\mathcal{F}\left(s_{i}^{n}\right) & \text { if }\left(\mathbf{f}_{\rho}\right)_{i+\frac{1}{2}}^{n}>0, \\
\mathcal{F}\left(s_{i+1}^{n}\right) & \text { otherwise, }\end{cases} \\
& \left\{\rho \mathcal{G}(\sigma) u_{1}\right\}_{i+\frac{1}{2}}^{n}=\left(\mathbf{f}_{\rho}\right)_{i+\frac{1}{2}}^{n} \times \begin{cases}\mathcal{G}\left(\sigma_{i}^{n}\right) & \text { if }\left(\mathbf{f}_{\rho}\right)_{i+\frac{1}{2}}^{n}>0, \\
\mathcal{G}\left(\sigma_{i+1}^{n}\right) & \text { otherwise, }\end{cases}
\end{aligned}
$$

where $\left(\mathbf{f}_{\rho}\right)_{i+\frac{1}{2}}^{n}$ is the first component of the numerical flux function (3.16).

Moreover, the following maximum principles are satisfied:

$$
s_{i}^{n+1} \geq \min \left(s_{i-1}^{n}, s_{i}^{n}, s_{i+1}^{n}\right), \quad \sigma_{i}^{n+1} \geq \min \left(\sigma_{i-1}^{n}, \sigma_{i}^{n}, \sigma_{i+1}^{n}\right),
$$

and

$$
\begin{aligned}
& \mathcal{F}\left(s_{i}^{n+1}\right) \leq \max \left(\mathcal{F}\left(s_{i-1}^{n}\right), \mathcal{F}\left(s_{i}^{n}\right), \mathcal{F}\left(s_{i+1}^{n}\right)\right), \\
& \mathcal{G}\left(\sigma_{i}^{n+1}\right) \leq \max \left(\mathcal{G}\left(\sigma_{i-1}^{n}\right), \mathcal{G}\left(\sigma_{i}^{n}\right), \mathcal{G}\left(\sigma_{i+1}^{n}\right)\right) .
\end{aligned}
$$

To prove the above theorem, we need the following three results where we establish, as soon as the Whitham condition (3.2) is satisfied, that the relaxation entropies decrease in $\mathrm{L}^{1}$-norm in the relaxation procedure. Its minimum will be seen to coincide with the equilibrium entropies. The relaxation entropies are thus compatible with the relaxation procedure in the sense of Chen, Levermore, and Liu 9].

The first result is devoted to the characteristic variables for the system (3.9). For the sake of clarity in the notation, we introduce the internal energy per direction:

$$
\rho e_{i j}=E_{i j}-\rho \frac{u_{i} u_{j}}{2}, \quad 1 \leq i \leq j \leq 2,
$$

to rewrite the pressure laws as follows:

$$
p_{i j}:=p_{i j}\left(\tau, e_{i j}\right)=2 \frac{e_{i j}}{\tau}, \quad 1 \leq i \leq j \leq 2,
$$

with $\tau=1 / \rho$.

Lemma 4.2. Let us set

$$
\begin{aligned}
& I=\pi_{11}+a^{2} \tau, \\
& X=e_{11}-\frac{\pi_{11}^{2}}{2 a^{2}}, \quad Y=e_{22}-\frac{\pi_{12}^{2}}{2 a^{2}}, \quad Z=e_{12}-\frac{\pi_{11} \pi_{12}}{2 a^{2}} .
\end{aligned}
$$

The weak solutions of (3.9) satisfy with no restrictive condition:

$$
\partial_{t} \rho \varphi(I, X, Y, Z)+\partial_{x} \rho \varphi(I, X, Y, Z) u_{1}=0,
$$

where $\varphi: \mathbb{R}^{4} \rightarrow \mathbb{R}$ denotes an arbitrary smooth function.

In the next statement, we propose entropies for the system (3.9) compatible with the equilibrium entropies (2.6) and (2.7). These relaxation entropies will be 
a function of the characteristic variables $(I, X, Y, Z)$ and defined over the following open subset of $\mathbb{R}^{4}$ :

$$
\begin{aligned}
\omega=\{ & (I, X, Y, Z) \in \mathbb{R}^{4} \\
& \forall\left(\tau, e_{11}, e_{22}, e_{12}\right) \in \mathbb{R}_{+}^{4}, a^{2} \tau-3 p_{11}\left(\tau, e_{11}\right)>0 \\
& I=p_{11}\left(\tau, e_{11}\right)+a^{2} \tau, X=e_{11}-\frac{1}{2 a^{2}} p_{11}\left(\tau, e_{11}\right)^{2} \\
& \left.Y=e_{22}-\frac{1}{2 a^{2}} p_{12}\left(\tau, e_{12}\right)^{2}, Z=e_{12}-\frac{1}{2 a^{2}} p_{11}\left(\tau, e_{11}\right) p_{12}\left(\tau, e_{12}\right)\right\}
\end{aligned}
$$

In fact, all points in $\omega$ coincide with an equilibrium state and satisfy the Whitham condition (3.2). Specifically, let us assume that the Riemann initial data (3.4) for the system (3.9) coincide with an equilibrium, i.e., $\left(\pi_{11}\right)_{L R}=\left(p_{11}\right)_{L R}$ and $\left(\pi_{12}\right)_{L R}=$ $\left(p_{12}\right)_{L R}$. Then for all points of the Riemann solution, the point $(I, X, Y, Z)$ defined by (4.8) belongs to $\omega$. Indeed, since the left and right states of the initial data are defined by equilibrium states, then $(I, X, Y, Z)_{L R}$ belongs to $\omega$. Now, since $(I, X, Y, Z)$ satisfies (4.9), it takes solely the values $(I, X, Y, Z)_{L}$ or $(I, X, Y, Z)_{R}$ and thus belongs to $\omega$. It is crucial to recall that, for the Riemann problem of (3.9), the relaxation procedure considers initial data which are at the equilibrium systematically. As a consequence, the above remark can be applied systematically. Across the relaxation procedure, always we consider points $(I, X, Y, Z)$ in $\omega$.

Lemma 4.3. Let $I, X, Y$, and $Z$, functions of $\left(\tau, e_{11}, e_{22}, e_{12}, \pi_{11}, \pi_{12}\right)$, be defined by (4.8). There exist unique functions $\bar{\tau}(I, X, Y, Z): \omega \rightarrow \mathbb{R}$ and $\bar{e}_{i j}(I, X, Y, Z)$ : $\omega \rightarrow \mathbb{R}$, with $1 \leq i \leq j \leq 2$, such that

$$
\begin{aligned}
& \left.\bar{\tau}(I, X, Y, Z)\right|_{\left\{\pi_{11}=p_{11}\left(\tau, e_{11}\right), \pi_{12}=p_{12}\left(\tau, e_{12}\right)\right\}}=\tau, \\
& \left.\bar{e}_{i j}(I, X, Y, Z)\right|_{\left\{\pi_{11}=p_{11}\left(\tau, e_{11}\right), \pi_{12}=p_{12}\left(\tau, e_{12}\right)\right\}}=e_{i j} .
\end{aligned}
$$

Let us set

$$
\bar{s}=p_{11}\left(\bar{\tau}, \bar{e}_{11}\right) \bar{\tau}^{3}, \quad \bar{\sigma}=\left(p_{11}\left(\bar{\tau}, \bar{e}_{11}\right) p_{22}\left(\bar{\tau}, \bar{e}_{2}\right)-p_{12}\left(\bar{\tau}, \bar{e}_{12}\right)^{2}\right) \bar{\tau}^{4} .
$$

Then the weak solutions of (3.9) satisfy

$$
\begin{aligned}
& \partial_{t} \rho \mathcal{F}(\bar{s})+\partial_{x} \rho \mathcal{F}(\bar{s}) u_{1}=0, \\
& \partial_{t} \rho \mathcal{G}(\bar{\sigma})+\partial_{x} \rho \mathcal{G}(\bar{\sigma}) u_{1}=0 .
\end{aligned}
$$

Let us note from now on that the linear degeneracy of the system (3.9) and the transport equations satisfied by $\mathcal{F}(\bar{s})$ and $\mathcal{G}(\bar{\sigma})$ imply that the Riemann solution of (3.9), detailed in Lemma 3.2, satisfies

$$
\begin{aligned}
& \mathcal{F}(\bar{s})(x, t)= \begin{cases}\mathcal{F}\left(\bar{s}_{L}\right), & \text { if } \frac{x}{t}<u_{1}^{\star}, \\
\mathcal{F}\left(\bar{s}_{R}\right), & \text { otherwise, }\end{cases} \\
& \mathcal{G}(\bar{\sigma})(x, t)= \begin{cases}\mathcal{G}\left(\bar{\sigma}_{L}\right), & \text { if } \frac{x}{t}<u_{1}^{\star}, \\
\mathcal{G}\left(\bar{\sigma}_{R}\right), & \text { otherwise. }\end{cases}
\end{aligned}
$$

In addition, if we assume that the left and right states are equilibrium states, then we have $\left(\bar{s}_{L}, \bar{s}_{R}\right)=\left(s_{L}, s_{R}\right)$ and $\left(\bar{\sigma}_{L}, \bar{\sigma}_{R}\right)=\left(\sigma_{L}, \sigma_{R}\right)$.

The last result concerns the minimum principle on the relaxation entropies: 
Lemma 4.4. Let $I, X, Y$, and $Z$, functions of $\left(\tau, e_{11}, e_{22}, e_{12}, \pi_{11}, \pi_{12}\right)$, be defined by (4.8) and let $\bar{s}$ and $\bar{\sigma}$ be defined by (4.13). Then

$$
\begin{aligned}
& \max _{\left(\pi_{11}, \pi_{12}\right) \in \mathbb{R}^{2}} \bar{s}=\left.\bar{s}\right|_{\left\{\pi_{11}=p_{11}\left(\tau, e_{11}\right), \pi_{12}=p_{12}\left(\tau, e_{12}\right)\right\}}=s, \\
& \max _{\left(\pi_{11}, \pi_{12}\right) \in \mathbb{R}^{2}} \bar{\sigma}=\left.\bar{\sigma}\right|_{\left\{\pi_{11}=p_{11}\left(\tau, e_{11}\right), \pi_{12}=p_{12}\left(\tau, e_{12}\right)\right\}}=\sigma .
\end{aligned}
$$

As a consequence, for all $\mathcal{F}$ and $\mathcal{G}$ which satisfy (2.8) and (2.9) respectively, the following minimum principles hold:

$$
\min _{\left(\pi_{11}, \pi_{12}\right) \in \mathbb{R}^{2}} \mathcal{F}(\bar{s})=\mathcal{F}(s) \quad \text { and } \quad \min _{\left(\pi_{11}, \pi_{12}\right) \in \mathbb{R}^{2}} \mathcal{G}(\bar{\sigma})=\mathcal{G}(\sigma) .
$$

Equipped with these three lemmas, we establish our main result.

Proof of Theorem 4.1. First, from the identities (3.11), (3.12), (3.13), and (3.14), the updated density $\rho_{i}^{n+1}$ reads:

$$
\rho_{i}^{n+1}=\frac{\Delta t}{\Delta x} \int_{0}^{\frac{\Delta x}{2 \Delta t}} \rho_{a_{i-\frac{1}{2}}}\left(\xi ; \mathbf{W}_{i-1}^{n}, \mathbf{W}_{i}^{n}\right) d \xi+\frac{\Delta t}{\Delta x} \int_{-\frac{\Delta x}{2 \Delta t}}^{0} \rho_{a_{i+\frac{1}{2}}}\left(\xi ; \mathbf{W}_{i}^{n}, \mathbf{W}_{i+1}^{n}\right) d \xi .
$$

Under the ordering condition (3.5), we have $\rho_{a}\left(\xi ; \mathbf{W}_{L}, \mathbf{W}_{R}\right)>0$. Then immediately we obtain the positiveness of $\rho_{i}^{n+1}$ for all $i \in \mathbb{Z}$. To conclude the proof of the first statement, (4.1), we have to establish the maximum principle, (4.7). Indeed, since the functions $\mathcal{F}$ and $\mathcal{G}$ satisfy (2.8) and (2.9), directly we deduce (4.6) from (4.7). Now, we have $\mathbf{w}_{i}^{n} \in \Omega$ which implies

$$
\min \left(s_{i-1}^{n}, s_{i}^{n}, s_{i+1}^{n}\right)>0 \quad \text { and } \quad \min \left(\sigma_{i-1}^{n}, \sigma_{i}^{n}, \sigma_{i+1}^{n}\right)>0 .
$$

As a consequence of (4.6), we obtain $s_{i}^{n+1}>0$ and $\sigma_{i}^{n+1}>0$, but for

$$
s_{i}^{n+1}=\frac{\left(p_{11}\right)_{i}^{n+1}}{\left(\rho_{i}^{n+1}\right)^{3}} \quad \text { and } \quad \sigma_{i}^{n+1}=\frac{\left(p_{11}\right)_{i}^{n+1}\left(p_{22}\right)_{i}^{n+1}-\left(\left(p_{12}\right)_{i}^{n+1}\right)^{2}}{\left(\rho_{i}^{n+1}\right)^{4}} .
$$

Arguing the positiveness of $\rho_{i}^{n+1}$, property (4.1) is proved.

To establish (4.7), we recall that, under the CFL restriction (3.10), the function $\mathbf{W}^{h}\left(x, t^{n}+\Delta t\right)$ denotes the solution at time $t^{n}+\Delta t$ of the Cauchy problem (3.9) for the piecewise constant initial data: the equilibrium state $\mathbf{W}_{i}^{n}$. With clear notation, we introduce $I^{h}, X^{h}, Y^{h}$, and $Z^{h}$ to define $\bar{s}^{h}$ according to the notation introduced in Lemma 4.3. We assume (2.8) and (2.9) to enforce the functions $\mathbf{w} \rightarrow \rho \mathcal{F}(s)$ and $\mathbf{w} \rightarrow \rho \mathcal{G}(\sigma)$ to be convex. We focus our attention on the function $\rho \mathcal{F}(s)$, while the maximum principle (4.7) for the function $\rho \mathcal{G}(\sigma)$ is obtained by similar arguments.

Arguing (3.14), a direct application of the well-known Jensen lemma gives

$$
\rho_{i}^{n+1} \mathcal{F}\left(s_{i}^{n+1}\right) \leq \frac{1}{\Delta x} \int_{x_{i-\frac{1}{2}}}^{x_{i+\frac{1}{2}}}\left\{\rho^{h} \mathcal{F}\left(s^{h}\right)\right\}\left(x, t^{n}+\Delta t\right) d x, \quad i \in \mathbb{Z} .
$$

Now, the minimum principle (4.17) gives

$$
\begin{aligned}
\rho_{i}^{n+1} \mathcal{F}\left(s_{i}^{n+1}\right) & \leq \frac{1}{\Delta x} \int_{x_{i-\frac{1}{2}}}^{x_{i+\frac{1}{2}}} \rho^{h} \min _{\left(\pi_{11}, \pi_{12}\right) \in \mathbb{R}^{2}} \mathcal{F}\left(\bar{s}^{h}\right)\left(x, t^{n}+\Delta t\right) d x \\
& \leq \frac{1}{\Delta x} \int_{x_{i-\frac{1}{2}}}^{x_{i+\frac{1}{2}}}\left\{\rho^{h} \mathcal{F}\left(\bar{s}^{h}\right)\right\}\left(x, t^{n}+\Delta t\right) d x, \quad i \in \mathbb{Z} .
\end{aligned}
$$


In addition, over each interval $I_{i}=\left[x_{i-\frac{1}{2}}, x_{i+\frac{1}{2}}\right)$ the functions $I^{h}, X^{h}, Y^{h}$, and $Z^{h}$ admit at most three values: $\left((I, X, Y, Z)_{i-1}^{n},(I, X, Y, Z)_{i}^{n},(I, X, Y, Z)_{i+1}^{n}\right)$, respectively. As a consequence, the function $\bar{s}^{h}$ over the interval $I_{i}$ admits at most three values: $s_{i-1}^{n}, s_{i}^{n}$ and $s_{i+1}^{n}$, which completes the proof of (4.7).

Next, we prove the discrete entropy inequality (4.2), while the second one, (4.3), is obtained by an easy adaptation. From Lemma 4.3, we know that the solution $\mathbf{W}^{h}$ of (3.9) satisfies

$$
\partial_{t} \rho^{h} \mathcal{F}\left(\bar{s}^{h}\right)+\partial_{x} \rho^{h} \mathcal{F}\left(\bar{s}^{h}\right) u_{1}^{h}=0 .
$$

By integration over $\left(x_{i-\frac{1}{2}}, x_{i+\frac{1}{2}}\right) \times\left(t^{n}, t^{n}+\Delta t\right)$, after a straightforward computation we obtain

$$
\begin{aligned}
\frac{1}{\Delta x} \int_{x_{i-\frac{1}{2}}}^{x_{i+\frac{1}{2}}}\left\{\rho^{h} \mathcal{F}\left(\bar{s}^{h}\right)\right\} & \left(x, t^{n+1}\right) d x-\rho_{i}^{n} \mathcal{F}\left(s_{i}^{n}\right) \\
+ & \frac{\Delta t}{\Delta x}\left(\left\{\rho \mathcal{F}(s) u_{1}\right\}_{i+\frac{1}{2}}^{n}-\left\{\rho \mathcal{F}(s) u_{1}\right\}_{i-\frac{1}{2}}^{n}\right)=0,
\end{aligned}
$$

where the numerical flux function $\left\{\rho \mathcal{F}(s) u_{1}\right\}_{i+\frac{1}{2}}^{n}$ is defined by (4.4). The sequence of inequalities (4.18)-(4.20) immediately gives the expected discrete entropy inequality (4.2). The proof of the theorem is thus concluded.

To conclude the present section, successively we prove the three intermediate lemmas.

Proof of Lemma 4.2, First, we assume that the solutions of system (3.9) is smooth enough. From the relaxation equations which govern $\rho u_{1}$ and $\rho u_{2}$, we deduce that

$$
\begin{aligned}
& \partial_{t} \rho \frac{u_{1}^{2}}{2}+\partial_{x} \rho \frac{u_{1}^{2}}{2} u_{1}+u_{1} \partial_{x} \pi_{11}=0, \\
& \partial_{t} \rho \frac{u_{2}^{2}}{2}+\partial_{x} \rho \frac{u_{2}^{2}}{2} u_{1}+u_{2} \partial_{x} \pi_{12}=0, \\
& \partial_{t} \rho \frac{u_{1} u_{2}}{2}+\partial_{x} \rho \frac{u_{1} u_{2}}{2} u_{1}+\frac{1}{2}\left(u_{2} \partial_{x} \pi_{11}+u_{1} \partial_{x} \pi_{12}\right)=0 .
\end{aligned}
$$

We subtract each relaxation kinetic energy $\rho \frac{u_{i} u_{j}}{2}$ from the associated relaxation total energy to obtain

$$
\begin{aligned}
& \partial_{t} \rho e_{11}+\partial_{x} \rho e_{11} u_{1}+\pi_{11} \partial_{x} u_{1}=0, \\
& \partial_{t} \rho e_{22}+\partial_{x} \rho e_{22} u_{1}+\pi_{12} \partial_{x} u_{1}=0, \\
& \partial_{t} \rho e_{12}+\partial_{x} \rho e_{12} u_{1}+\frac{1}{2}\left(\pi_{11} \partial_{x} u_{2}+\pi_{12} \partial_{x} u_{1}\right)=0 .
\end{aligned}
$$

Arguing the continuity equation $\partial_{t} \rho+\partial_{x} \rho u_{1}=0$, we rewrite these three equations above as

$$
\begin{aligned}
& \partial_{t} e_{11}+u_{1} \partial_{x} e_{11}+\frac{\pi_{11}}{\rho} \partial_{x} u_{1}=0 \\
& \partial_{t} e_{22}+u_{1} \partial_{x} e_{22}+\frac{\pi_{12}}{\rho} \partial_{x} u_{1}=0 \\
& \partial_{t} e_{12}+u_{1} \partial_{x} e_{12}+\frac{1}{2}\left(\frac{\pi_{11}}{\rho} \partial_{x} u_{2}+\frac{\pi_{12}}{\rho} \partial_{x} u_{1}\right)=0
\end{aligned}
$$


In addition, the approximate relaxation pressures $\pi_{11}$ and $\pi_{12}$ satisfy

$$
\begin{aligned}
& \partial_{t} \pi_{11}+u_{1} \partial_{x} \pi_{11}+\frac{a^{2}}{\rho} \partial_{x} u_{1}=0, \\
& \partial_{t} \pi_{12}+u_{1} \partial_{x} \pi_{12}+\frac{a^{2}}{\rho} \partial_{x} u_{2}=0,
\end{aligned}
$$

which gives, after multiplied by $\pi_{11} / a^{2}$ and $\pi_{12} / a^{2}$, respectively,

$$
\begin{aligned}
& \partial_{t} \frac{\pi_{11}^{2}}{2 a^{2}}+u_{1} \partial_{x} \frac{\pi_{11}^{2}}{2 a^{2}}+\frac{\pi_{11}}{\rho} \partial_{x} u_{1}=0, \\
& \partial_{t} \frac{\pi_{12}^{2}}{2 a^{2}}+u_{1} \partial_{x} \frac{\pi_{12}^{2}}{2 a^{2}}+\frac{\pi_{12}}{\rho} \partial_{x} u_{2}=0, \\
& \partial_{t} \frac{\pi_{11} \pi_{12}}{2 a^{2}}+u_{1} \partial_{x} \frac{\pi_{11} \pi_{12}}{2 a^{2}}+\frac{1}{2}\left(\frac{\pi_{11}}{\rho} \partial_{x} u_{2}+\frac{\pi_{12}}{\rho} \partial_{x} u_{1}\right)=0 .
\end{aligned}
$$

We immediately have

$$
\partial_{t} X+u_{1} \partial_{x} X=0, \quad \partial_{t} Y+u_{1} \partial_{x} Y=0, \quad \partial_{t} Z+u_{1} \partial_{x} Z=0 .
$$

Moreover, from the relaxation continuity equation, we deduce

$$
\partial_{t} \tau+u_{1} \partial_{x} \tau-\frac{1}{\rho} \partial_{x} u_{1}=0, \quad \tau=\frac{1}{\rho}
$$

to easily obtain

$$
\partial_{t} I+u_{1} \partial_{x} I=0 .
$$

Now, let us consider a smooth function $\varphi: \mathbb{R}^{4} \rightarrow \mathbb{R}$. We deduce from (4.21) and (4.22) the transport equation

$$
\partial_{t} \varphi(I, X, Y, Z)+u_{1} \partial_{x} \varphi(I, X, Y, Z)=0,
$$

to obtain the expected conservation equation (4.9). Since the system (3.9) only admits linearly degenerate fields, the weak solutions of (3.9) also satisfy (4.9).

Proof of Lemma 4.3. For the sake of simplicity in the notation, we set $M=$ $(I, X, Y, Z)$. For all $M \in \omega$, we search functions $\bar{\tau}: \omega \rightarrow \mathbb{R}$ and $\bar{e}_{i j}: \omega \rightarrow \mathbb{R}$ which satisfy

$$
\left\{\begin{array}{l}
I=p_{11}\left(\bar{\tau}(M), \bar{e}_{11}(M)\right)+a^{2} \bar{\tau}(M), \\
X=\bar{e}_{11}(M)-\frac{1}{2 a^{2}} p_{11}\left(\bar{\tau}(M), \bar{e}_{11}(M)\right)^{2}, \\
Y=\bar{e}_{22}(M)-\frac{1}{2 a^{2}} p_{12}\left(\bar{\tau}(M), \bar{e}_{12}(M)\right)^{2}, \\
Z=\bar{e}_{12}(M)-\frac{1}{2 a^{2}} p_{11}\left(\bar{\tau}(M), \bar{e}_{11}(M)\right) p_{12}\left(\bar{\tau}(M), \bar{e}_{12}(M)\right) .
\end{array}\right.
$$

By definition of $\omega \subset \mathbb{R}^{4}$, for all $M \in \omega$ the above system admits a solution which satisfies the Whitham condition (3.2). Now, this solution is shown to define a unique function. To simplify the notation, we omit the dependence on $M$.

First, we focus on $\bar{\tau}$ and $\bar{e}_{11}$. We write

$$
p_{11}\left(\bar{\tau}, \bar{e}_{11}\right)=I-a^{2} \bar{\tau}
$$

and then

$$
\bar{e}_{11}=X+\frac{1}{2 a^{2}}\left(I-a^{2} \bar{\tau}\right)^{2},
$$


to obtain

$$
I=a^{2} \bar{\tau}+p_{11}\left(\bar{\tau}, X+\frac{1}{2 a^{2}}\left(I-a^{2} \bar{\tau}\right)^{2}\right):=\Theta_{\tau}(\bar{\tau})
$$

Moreover, the function $\Theta_{\tau}$ is increasing:

$$
\Theta_{\tau}^{\prime}(\bar{\tau})=\frac{1}{\bar{\tau}}\left(a^{2} \bar{\tau}-3 p_{11}\left(\bar{\tau}, \bar{e}_{11}\right)\right)>0
$$

which concludes the existence and uniqueness of $\bar{\tau}$ but also $\bar{e}_{11}$ by (4.24). In addition, we note that $\bar{\tau}$ and $\bar{e}_{11}$ only depend on $(I, X)$ and not on $(Y, Z)$.

Concerning $\bar{e}_{22}$ and $\bar{e}_{12}$, we have

$$
\begin{aligned}
& \bar{e}_{22}(M)=Y+\frac{1}{2 a^{2}} p_{12}\left(\bar{\tau}(I, X), \bar{e}_{12}(M)\right)^{2} \\
& Z:=\Theta_{e_{12}}\left(\bar{e}_{12}\right) \\
& \quad=\bar{e}_{12}(M)-\frac{1}{2 a^{2}} p_{11}\left(\bar{\tau}(I, X), \bar{e}_{11}(I, X)\right) p_{12}\left(\bar{\tau}(I, X), \bar{e}_{12}(M)\right),
\end{aligned}
$$

where the function $\Theta_{e_{12}}$ is increasing:

$$
\Theta_{e_{12}}^{\prime}\left(\bar{e}_{12}\right)=\frac{1}{a^{2} \bar{\tau}}\left(a^{2} \bar{\tau}-p_{11}\left(\bar{\tau}, \bar{e}_{11}\right)\right)>0,
$$

and then we have the existence and uniqueness of $\bar{e}_{12}$ but also $\bar{e}_{22}$.

Now, with $\left(\tau, e_{11}, e_{22}, e_{12}\right)$ fixed, we show that $\bar{\tau}=\tau$ and $\bar{e}_{i j}=e_{i j}, 1 \leq i \leq j \leq 2$, as soon as the equilibrium is reached: $\pi_{11}=p_{11}\left(\tau, e_{11}\right)$ and $\pi_{12}=p_{12}\left(\tau, e_{12}\right)$. Arguing the definition of $X$ and $I$, given by (4.8), the equation $\Theta_{\tau}(\bar{\tau})-I=0$ is rewritten as

$$
\Theta_{\tau}^{e q}(\bar{\tau})=0
$$

with

$$
\Theta_{\tau}^{e q}(\bar{\tau})=a^{2}(\bar{\tau}-\tau)+p_{11}\left(\bar{\tau}, e+\frac{a^{2}}{2}(\bar{\tau}-\tau)^{2}+p_{11}\left(\tau, e_{11}\right)(\tau-\bar{\tau})\right)-p_{11}\left(\tau, e_{11}\right),
$$

where $\tau$ is the unique solution since the function $\Theta_{\tau}^{e q}$ is increasing:

$$
\left(\Theta_{\tau}^{e q}\right)^{\prime}(\bar{\tau})=\Theta_{\tau}^{\prime}(\bar{\tau})
$$

Moreover, since at the equilibrium we have $\bar{\tau}=\tau$, from the identities (4.24), (4.25), and (4.26) we immediately deduce $\bar{e}_{i j}=e_{i j}$ with $1 \leq i \leq j \leq 2$.

To conclude the proof, we recall that all functions of $M$ satisfy (4.9). We note that $\bar{s}$ and $\bar{\sigma}$ are functions of the variable $M$ and defined by (4.13). As a consequence, we immediately deduce that the functions $M \rightarrow \mathcal{F}(\bar{s}(M))$ and $M \rightarrow \mathcal{G}(\bar{\sigma}(M))$ satisfy the expected equations (4.14) and (4.15), respectively.

Proof of Lemma 4.4. The proof is obtained after the computation of the derivatives of the function $\bar{s}$ and $\bar{\sigma}$ understood as the function of $\left(\tau, e_{11}, e_{22}, e_{12}, \pi_{11}, \pi_{12}\right)$ when $I, X, Y$, and $Z$ are defined by (4.8). We skip this easy but long computation, and we give the following result:

$$
\begin{aligned}
\frac{\partial \bar{s}}{\partial \pi_{11}} & =2 \frac{p_{11}\left(\bar{\tau}, \bar{e}_{11}\right)-\pi_{11}}{a^{2}\left(a^{2} \bar{\tau}-3 p_{11}\left(\bar{\tau}, \bar{e}_{11}\right)\right)} \bar{\tau}^{3}, \quad \frac{\partial \bar{s}}{\partial \pi_{12}}=0 \\
\frac{\partial \bar{\sigma}}{\partial \pi_{11}} & =\frac{2 \bar{\tau}^{3}}{a^{2}}\left(p_{22}\left(\bar{\tau}, \bar{e}_{22}\right)\left(p_{11}\left(\bar{\tau}, \bar{e}_{11}\right)-\pi_{11}\right)+p_{12}\left(\bar{\tau}, \bar{e}_{12}\right)\left(p_{12}\left(\bar{\tau}, \bar{e}_{12}\right)-\pi_{12}\right)\right) \\
\frac{\partial \bar{\sigma}}{\partial \pi_{12}} & =\frac{2 \bar{\tau}^{3}}{a^{2}}\left(p_{12}\left(\bar{\tau}, \bar{e}_{12}\right) \pi_{11}-p_{11}\left(\bar{\tau}, \bar{e}_{11}\right) \pi_{12}\right) .
\end{aligned}
$$


We immediately obtain that the equilibrium manifold $\left\{\pi_{11}=p_{11}\left(\tau, e_{11}\right), \pi_{12}=\right.$ $\left.p_{12}\left(\tau, e_{12}\right)\right\}$ defines the unique extrema of the functions $\bar{s}$ and $\bar{\sigma}$. By computing the second order derivatives of $\bar{s}$ and $\bar{\sigma}$, we establish that these extrema are, in fact, maximum.

\section{Numerical Results}

In this section, we perform numerical experiments obtained with the relaxation scheme (3.15)-(3.16). We propose to consider several Riemann problems over the interval $(-0.5,0.5)$, the initial discontinuity being located at $x=0$. We use a uniform mesh made of 500 cells. The CFL number fixed to 0.5 according to the CFL condition (3.10). All the results we display are systematically compared with the exact Riemann solution and the approximate solution performed with the HLLE scheme (see [16]) and the Lax-Friedrichs scheme (see [14, 18]), except for the last test.

The first test is characterized by the following initial data:

\begin{tabular}{|l|l|l|l|l|l|l|}
\hline & $\rho$ & $u_{1}$ & $u_{2}$ & $p_{11}$ & $p_{12}$ & $p_{22}$ \\
\hline left state & 1 & 0 & 0 & 2 & 0.05 & 0.6 \\
\hline right state & 0.125 & 0 & 0 & 0.2 & 0.1 & 0.2 \\
\hline
\end{tabular}

This test is the classical Sod shock tube. The numerical results for $t=0.125$ are displayed in Figure 1. The numerical solutions obtained with the HLLE scheme and the relaxation scheme have the same accuracy, while the Lax-Friedrichs scheme
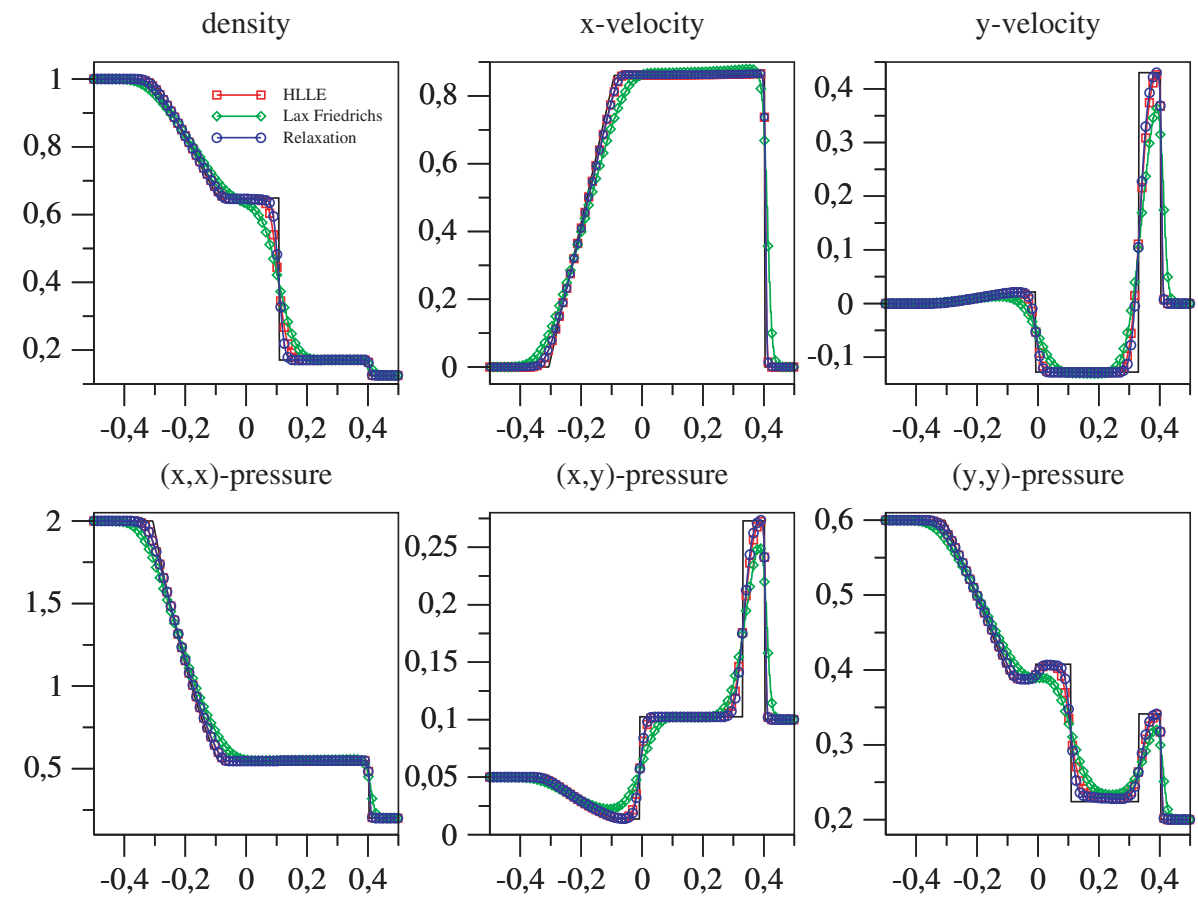

Figure 1. Sod tube problem: exact solution (fill line), relaxation scheme (o symbol), HLLE scheme ( $\square$ symbol), and Lax-Friedrichs scheme $(\diamond$ symbol). 
gives a very diffusive approximation. We note a better accuracy for the relaxation scheme when approximating the contact wave. This is emphasized by the following table where, we give the $l^{1}$-norm of the error in $\%$ :

\begin{tabular}{|c|l|l|l|l|l|l|}
\hline & $\rho$ & $u_{1}$ & $u_{2}$ & $p_{11}$ & $p_{12}$ & $p_{22}$ \\
\hline Relaxation & 0.75 & 0.73 & 1.01 & 0.89 & 0.38 & 0.60 \\
\hline HLLE & 1.08 & 0.70 & 1.23 & 0.86 & 0.45 & 0.78 \\
\hline Lax-Friedrichs & 1.70 & 1.66 & 1.65 & 1.98 & 0.66 & 1.14 \\
\hline
\end{tabular}

In the second test, we consider the following initialization:

\begin{tabular}{|l|l|l|l|l|l|l|}
\hline & $\rho$ & $u_{1}$ & $u_{2}$ & $p_{11}$ & $p_{12}$ & $p_{22}$ \\
\hline left state & 1 & 1 & 1 & 1 & 0 & 1 \\
\hline right state & 1 & -1 & -1 & 1 & 0 & 1 \\
\hline
\end{tabular}

The exact solution is made of two shock waves separated by a contact discontinuity. The numerical approximations for $t=0.125$ are displayed in Figure 2, Both HLLE and relaxation schemes give accurate solutions. However, we observe a larger spike for the relaxation approximation in the 2-contact discontinuity for the density and the pressure $p_{22}$. Let us note that these spikes are standard in the framework of the classical Euler equations. Indeed, not only the relaxation scheme but also the Roe scheme or the Osher scheme (for instance) involve the same spikes when approximating shocks resulting from two opposing hypersonic flows (see Liska and Wendroff [21] or Noh [24]). This difficulty is preserved in the present framework.

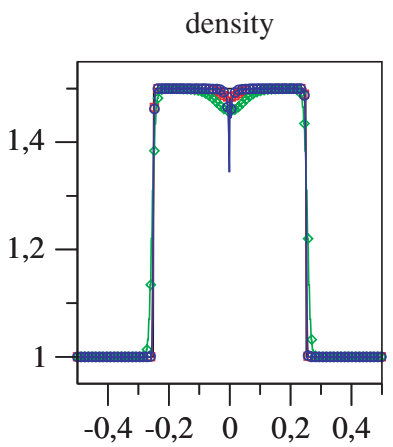

$(\mathrm{x}, \mathrm{x})$-pressure

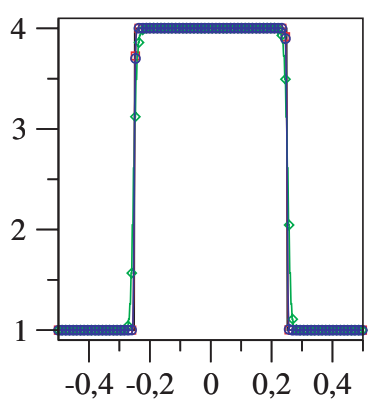

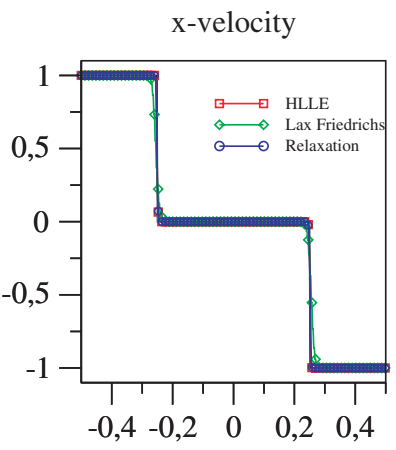

$(\mathrm{x}, \mathrm{y})$-pressure

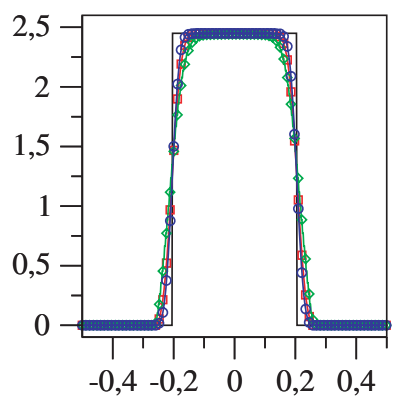

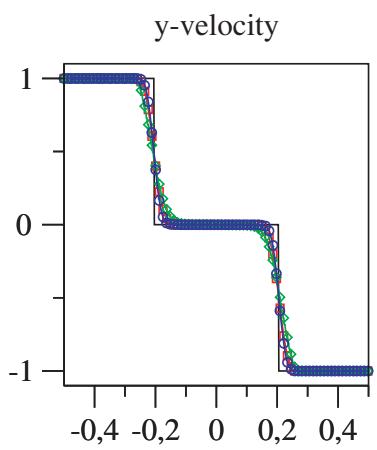

$(\mathrm{y}, \mathrm{y})$-pressure

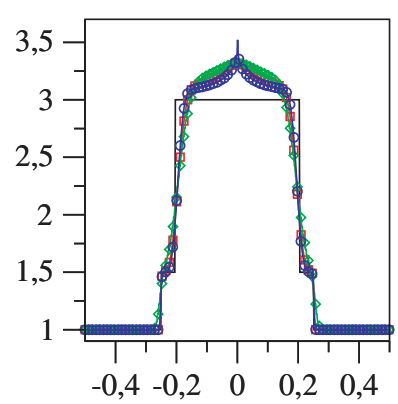

Figure 2. Two shock waves problem: exact solution (fill line), relaxation scheme (o symbol), HLLE scheme ( $\square$ symbol), and LaxFriedrichs scheme ( $\diamond$ symbol). 
The next test is obtained with the following left and right states:

\begin{tabular}{|l|l|l|l|l|l|l|}
\hline & $\rho$ & $u_{1}$ & $u_{2}$ & $p_{11}$ & $p_{12}$ & $p_{22}$ \\
\hline left state & 2 & -0.5 & -0.5 & 1.5 & 0.5 & 1.5 \\
\hline right state & 1 & 1 & 1 & 1 & 0 & 1 \\
\hline
\end{tabular}

In contrast with the previous test, at this time the exact solution is made of two rarefaction waves. The numerical approximations for $t=0.15$ are displayed in Figure 3. Once again, HLLE and relaxation schemes give approximate solutions in good agreement with the exact solution. However, both schemes do not predict accurate intermediate states for the pressure $p_{22}$ with this level of mesh refinement. Of course, good prediction of $p_{22}$ is obtained as soon as the mesh is fine enough.

The last numerical test is devoted to improve the robustness of the relaxation scheme. Indeed, the initial data is not assumed to belong to the hyperbolic domain $\Omega$. However, the numerical results displayed in Figure 4 show that the approximate solutions obtained by the relaxation scheme seem to converge to a solution of the initial system. We consider the following left and right states:

\begin{tabular}{|l|l|l|l|l|l|l|}
\hline & $\rho$ & $u_{1}$ & $u_{2}$ & $p_{11}$ & $p_{12}$ & $p_{22}$ \\
\hline left state & 2 & 1.05 & 0 & -0.205 & 0.05 & 0.6 \\
\hline right state & 0.125 & 0 & 0 & 0.2 & 0.1 & 0.2 \\
\hline
\end{tabular}

where we note that the left pressure $p_{11}$ is negative. Actually, severe numerical simulations (for instance, see Berthon and Dubroca [6]) involve physics which impose

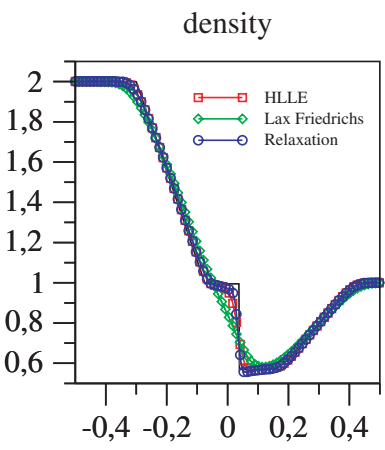

$(\mathrm{x}, \mathrm{X})$-pressure

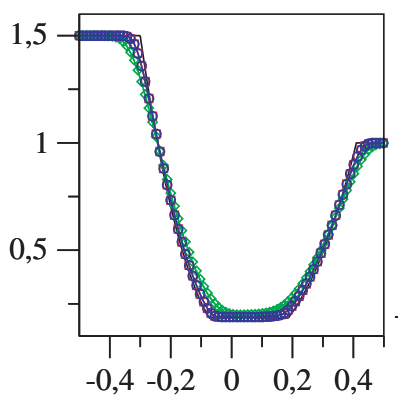

x-velocity

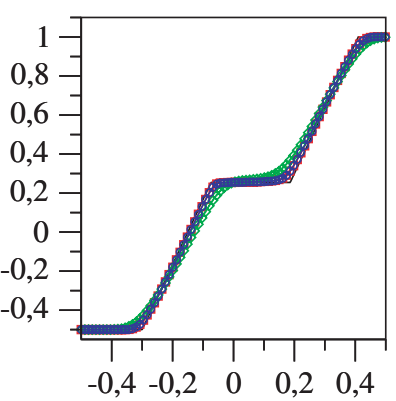

$(\mathrm{x}, \mathrm{y})$-pressure

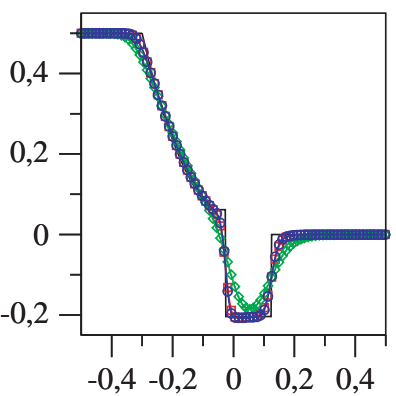

y-velocity

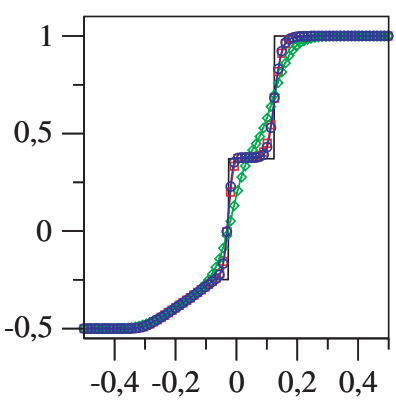

$(\mathrm{y}, \mathrm{y})$-pressure

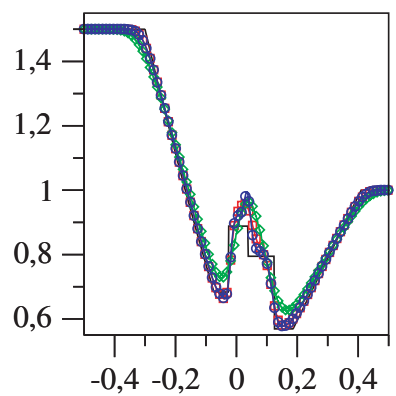

Figure 3. Two rarefaction waves problem: exact solution (fill line), relaxation scheme (o symbol), HLLE scheme ( $\square$ symbol), and Lax-Friedrichs scheme $(\diamond$ symbol). 

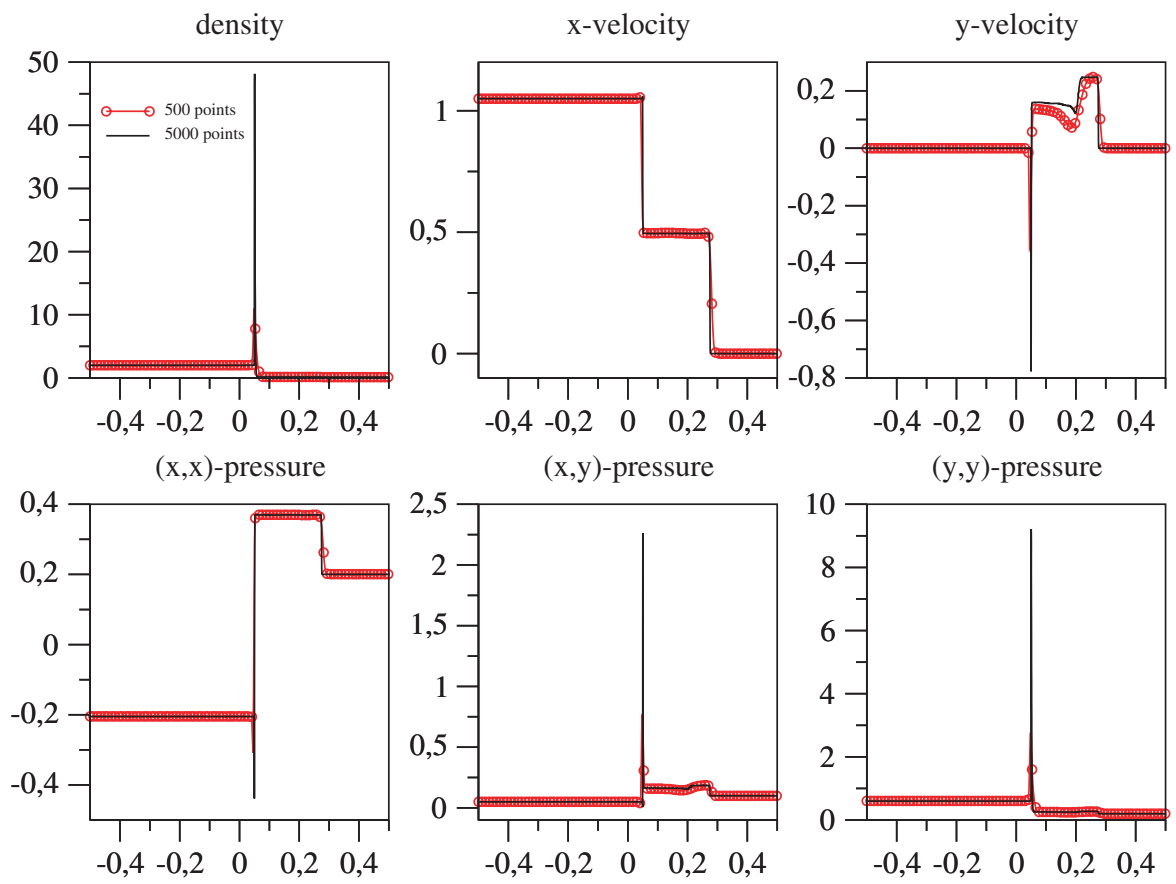

FiguRE 4. Nonhyperbolic problem: approximate solutions obtained with the relaxation scheme for a mesh made of 500 cells (o symbol) and 5000 cells (fill line).

that the trace of the pressure tensor is positive but do not impose a positive pressure tensor. In Figure 5, we observe that the trace of the pressure tensor remains positive during the simulation. Of course, since the pressure $p_{11}$ is negative, both HLLE and Lax-Friedrichs schemes do not iterate. In Figure 4, we just display at time $t=0.1$ the numerical solution obtained with the relaxation scheme where two levels of mesh refinement are considered; respectively 500 and 5000 cells.

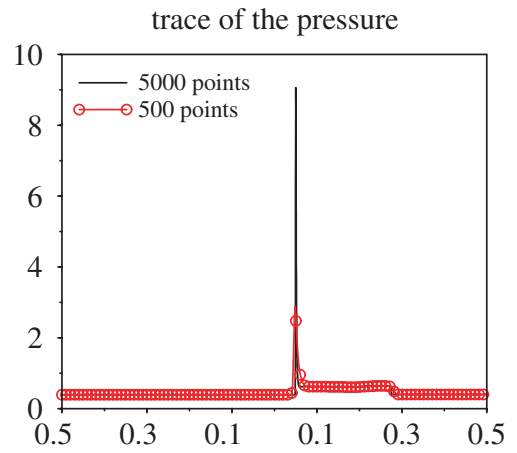

Figure 5. Trace of the pressure tensor in the case of the nonhyperbolic problem. 


\section{ACKNOWLEDGMENTS}

The author is thankful for helpful discussions with P. Charrier and B. Dubroca.

\section{REFERENCES}

1. D. Aregba-Driollet and R. Natalini, Convergence of relaxation schemes for conservation laws, Appl. Anal., 61 (1996), Nos. 1-2, 163-190. MR1625520

2. M. Baudin, C. Berthon, F. Coquel, R. Masson, and Q. H. Tran, A relaxation method for twophase flow models with hydrodynamic closure law, Num. Math., 99 (2005), No.3, 411-440. MR:2117734 (2005h:76079)

3. C. Berthon, Inégalités d'entropie pour un schéma de relaxation, C. R. Acad. Sci. Paris, 340 (2005), No.1, 63-68. MR.2112043

4. C. Berthon, B. Braconnier, and B. Nkonga, Numerical approximation of a degenerated non conservative multifluid model: relaxation scheme, Int. J. Numer. Methods Fluids, 48 (2005), No.1, 85-90.

5. C. Berthon, M. Breuss, and M.-O. Titeux, A relaxation scheme for the approximation of the pressureless Euler equations, Numer. Methods Partial Diff. Eq., 22 (2006), 484-505.

6. C. Berthon and B. Dubroca, work in preparation.

7. F. Bouchut, Entropy satisfying flux vector splittings and kinetic BGK models, Numer. Math., 94 (2003), No. 4, 623-672. MR1990588 (2005e:65129)

8. C. Chalons and F. Coquel, Navier-Stokes equations with several independent pressure laws and explicit predictor-corrector schemes, preprint.

9. G.Q. Chen, C.D. Levermore, and T.P. Liu, Hyperbolic Conservation Laws with Stiff Relaxation Terms and Entropy, Comm. Pure Appl. Math., 47 (1995), 787-830. MR1280989 (95h:35133)

10. F. Coquel, E. Godlewski, A. In, B. Perthame, and P. Rascle, Some new Godunov and relaxation methods for two phase flows, Proceedings of an International Conference on Godunov Methods: Theory and Applications, Kluwer Academic/Plenum Publishers, 2002. MR1963591 (2004a:76093)

11. F. Coquel and B. Perthame, Relaxation of Energy and Approximate Riemann Solvers for General Pressure Laws in Fluid Dynamics, SIAM J. Numer. Anal., 35 (1998), 6, 2223-2249. MR 1655844 (2000a:76129)

12. B. Dubroca, M. Tchong, P. Charrier, V.T. Tikhonchuk, and P.-M. Morreeuw, Magnetic field generation in plasma due to anisotropic laser heating, J. Phys. Plasma, accepted.

13. T. I. Gombosi, C. P. T. Groth, P. L. Roe, and S. L. Brown, 35-Moment closure for rarefied gases: Derivation, transport equations, and wave structure, preprint.

14. E. Godlewski and P.A. Raviart, Numerical Approximation of Hyperbolic System of Conservation Laws, Applied Mathematical Sciences, 118, Springer, New York, 1996. MR.1410987 (98d:65109)

15. H. Grad, On the kinetic theory of rarefied gas, Comm. Pure Appl. Math., 2 (1949), 331-407. MR0033674 (11:473a)

16. A. Harten, P.D. Lax, and B. van Leer, On Upstream Differencing and Godunov-Type Schemes for Hyperbolic Conservation Laws, SIAM Review, 25 (1983), 35-61. MR0693713 (85h:65188)

17. S. Jin and Z. Xin, The Relaxation Scheme for Systems of Conservation Laws in Arbitrary Space Dimension, Comm. Pure Appl. Math., 45 (1995), 235-276. MR1322811 (96c:65134)

18. R. J. LeVeque, Finite volume methods for hyperbolic problems, Cambridge Texts in Applied Mathematics. Cambridge University Press, Cambridge, 2002. MR1925043 (2003h:65001)

19. C.D. Levermore, Moment closure hierarchies for kinetic theory, J. Statist. Phys., 83 (1996), 1021-1065. MR1392419(97e:82041)

20. C.D. Levermore and W.J. Morokoff, The Gaussian moment closure for gas dynamics, SIAM J. Appl. Math., 59 (1998), 1, 72-96. MR.1641154 (99f:76114)

21. R. Liska and B. Wendroff, Comparison of several difference schemes on $1 D$ and $2 D$ test problems for the Euler equations, SIAM J. Sci. Comput., 25 (2003), No.3, 995-1017. MR2046122 (2004k:76088)

22. T.P. Liu, Hyperbolic conservation laws with relaxation, Comm. Math. Phys., 108 (1987), 153-175. MR0872145 (88f:35092)

23. R. Natalini, Convergence to equilibrium for the relaxation approximation of conservation laws, Comm. Pure Appl. Math., 49 (1996), 1-30. MR1391756 (97c:35131) 
24. W.F. Noh, Errors for calculations of strong shocks using an artificial viscosity and an artificial heat flux, J. Comput. Phys., 72 (1987), 78-120.

25. I. Suliciu, Energy estimates in rate-type thermo-viscoplasticity, Int. J. Plast., 14 (1998), 227244.

26. J. Whitham, Linear and Nonlinear Waves, Wiley, New York, 1974. MR0483954 (58:3905)

MAB, UMR 5466 CNRS, Université Bordeaux 1, 351 Cours de la libÉration, 33400 Talence, France

E-mail address: Christophe.Berthon@math.u-bordeaux1.fr 